Arkivoc

Free to Authors and Readers
A Platinum Open Access Journal for Organic Chemistry

Review

DOAJ Seal
Arkivoc 2021, part iii, 157-184

\title{
Asymmetric organic synthesis with stoichiometric transition metal complexes
}

\author{
Helena C. Malinakova \\ Department of Chemistry, The University of Kansas, 1140 Gray-Little Hall, 1567 Irving Hill Road, \\ Lawrence, KS 66045, USA \\ Email: hmalinakova@ku.edu
}

Dedicated to Professor Lanny S. Liebeskind for his outstanding contribution to organometallic chemistry

Received 12-15-2020

Accepted 02-16-2021

Published on line 03-05-2021

\section{Abstract}

Significant recent contributions to the advancement of asymmetric organic synthesis featuring transformations of stoichiometric transition metal complexes are described. Studies that led to the development of powerful synthetic methodologies, as well as projects aimed at elucidation of relevant fundamental concepts, are included. A particular emphasis is placed on applications of complexes with planar chirality.<smiles>C[C@H]1C(=O)O[N+]2(c3cc(Cl)ccc3[N+]3(Cc4ccccc4)N4CCC[C@H]4C(=O)N32)N1Cc1ccc(Cl)c(Cl)c1</smiles>

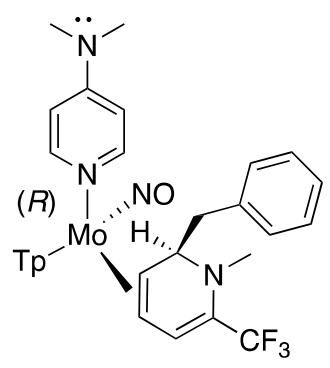<smiles>C[C@@H]1C(=O)ON(C(=O)O)[C@H]1C</smiles><smiles>CCOC1CN=[V]c2ccccc2P1P(P)P</smiles>

Keywords: Stoichiometric transition metal-complexes, planar chirality, metal-mediated dearomatization, metal-mediated $\mathrm{C}-\mathrm{H}$ activation, nucleophilic attack on polyhapto-coordinated ligands, asymmetric synthesis of heterocycles 


\section{Table of Contents}

1. Introduction

2. Methods for Stereoselective $\mathrm{C}-\mathrm{H}$ Functionalization and Relevant Mechanistic Insights

2.1. C-H Functionalization in $\mathrm{Ni}$-complexes

2.2. C-H Functionalization in Co-complexes

2.3. $\mathrm{C}-\mathrm{H}$ Functionalization in $\mathrm{Cr}$-arene complexes

2.4. Complexes with a Pd-bonded stereogenic carbon

3. Methods for Elaboration of Organometallic Complexes with Planar Chirality

3.1. Elaboration of $\mathrm{Cr}-$, Fe- and Ru-complexes with planar chirality

3.2. Elaboration of Mo-Complexes with Planar Chirality

3.3. Dearomatization via $\eta 2$-Coordination with $\mathrm{W}$ and $\mathrm{Mo}$

4. Conclusions

References

\section{Introduction}

Discovery of the profound effects that bonding to a transition metal has on the reactivity of an organic molecule ${ }^{1}$ initiated the rapid rise of a new field known as organometallic chemistry. In many areas, the early studies with stoichiometric models of transition metal complexes ${ }^{2,3}$ led rapidly to the development of powerful Nobel Prize-winning transition metal-catalyzed reactions. ${ }^{4}$ Notwithstanding the success of catalytic transformations, an alternative approach to new reaction development, employing stoichiometric quantities of transition metal complexes, has made significant contributions to the advancement of organic synthesis. 5,6

Systematic studies of stoichiometric transition metal complexes by researchers with insights into both the fundamentals of organometallic chemistry, as well as the needs and challenges of synthetic organic chemistry and the pharmaceutical industry, have led to original discoveries and the development of synthetic tools that would have never been realized if all research efforts were focused exclusively on transformations easily amenable to a catalytic turnover.

The aim of the present review is to highlight contributions to the advancement of asymmetric organic synthesis featuring transformations of stoichiometric transition metal complexes. The review includes work published over approximately the past fifteen years. Although the main emphasis is placed on studies that led to the development of powerful synthetic methodologies, examples of experiments with stoichiometric transition metal complexes designed to elucidate important fundamental concepts are included.

The material is divided into two main thematic areas. In the first area, a complexation between the transition metal and the organic fragment creates a unique chiral nonracemic organometallic scaffold that facilitates a stereoselective $\mathrm{C}-\mathrm{H}$ functionalization of the organic substrate ("stereoselective $\mathrm{C}-\mathrm{H}$ functionalization").

In the second area, the transition metal centre serves to differentiate two enantiotopic faces of an organic substrate for further elaboration via nucleophilic or electrophilic attack on the transition metalbonded organic ligand ("elaboration of templates with planar chirality"). 


\section{Methods for Stereoselective C-H Functionalization and Relevant Mechanistic Insights}

\subsection{C-H Functionalization in $\mathrm{Ni}$-complexes}

An imaginative application of stoichiometric complexes of $\mathrm{Ni}(\mathrm{II})$ has been devised to realize asymmetric synthesis of $\alpha, \alpha$-disubstituted amino acids. ${ }^{7} \alpha$-(Methyl)cysteine, a rare naturally occurring amino acid that shows promising antitumor activity, was successfully targeted by this methodology. The protocol relies on diastereoselective alkylation of a $\mathrm{Ni}$ (II) complex with a Schiff base-derived chiral ligand synthesized from an enantiopure ketone backbone and a racemic amino acid (alanine) (Scheme 1). Two types of the enantiopure backbones featuring either axial chirality or a proline-derived scaffold were applied to construct the $\mathrm{Ni}(\mathrm{II})$ templates (Scheme 1).
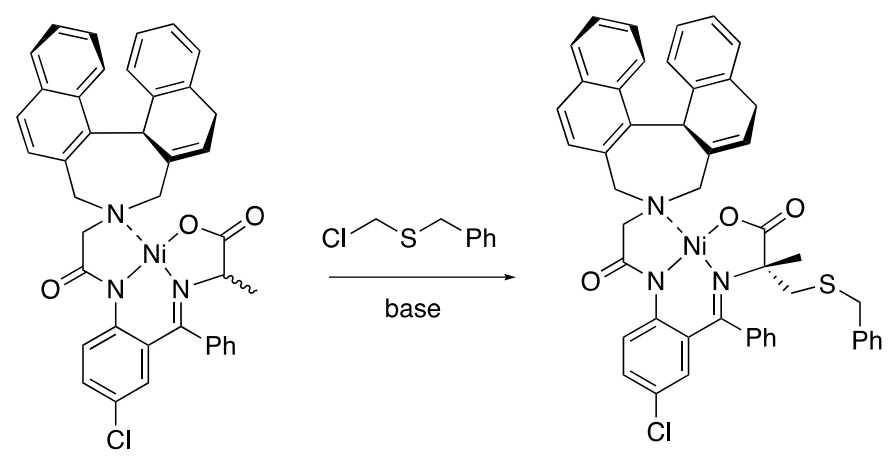

$$
\operatorname{dr} 89: 11
$$
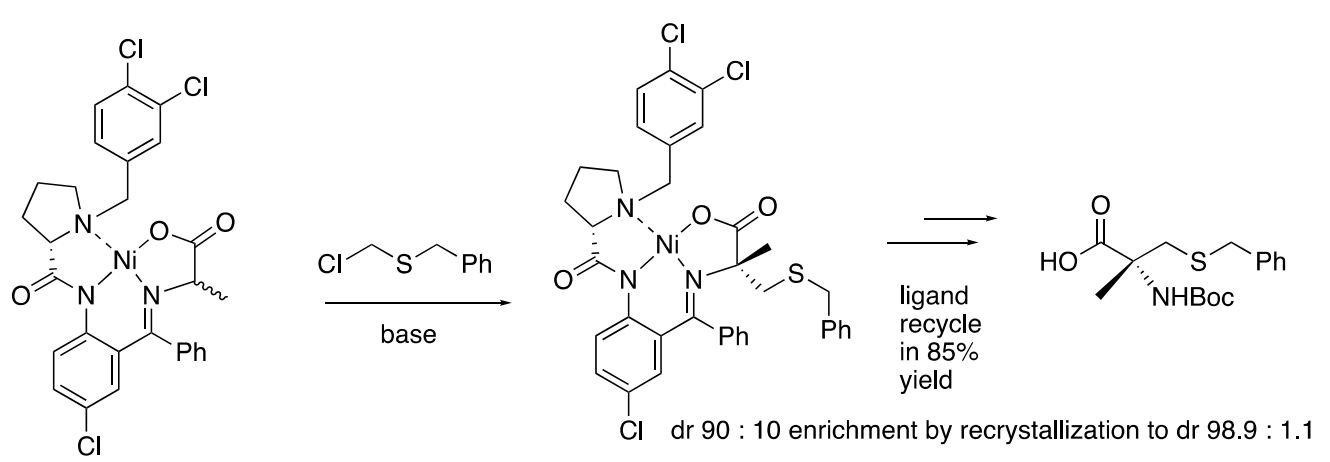

\section{Scheme 1}

The treatment of the $\mathrm{Ni}(\mathrm{II})$ scaffolds with base and S-benzylthiomethylchloride afforded under optimized conditions the elaborated $\mathrm{Ni}$ (II) complexes in approximately $9: 1$ diastereoselectivity, favoring the diastereomer with $(R)$ configuration at the $\alpha$-carbon of the quaternary amino acid. The release of the free amino acid, as well as recycling of the chiral ligand in $85 \%$ yield, has been demonstrated, although the enantiopurity of the quaternary amino acid was not verified. ${ }^{5}$ This methodology has been successfully applied to the construction of amino acids featuring unsaturated functionalities, ${ }^{8}$ that are of interest for their biological activities applicable to drug development efforts (Scheme 2). The study is notable for demonstrating the feasibility of Cu-catalyzed oxidative Glaser cross-coupling of terminal alkynes groups present on $\mathrm{Ni}(\mathrm{II})$ scaffolds. 

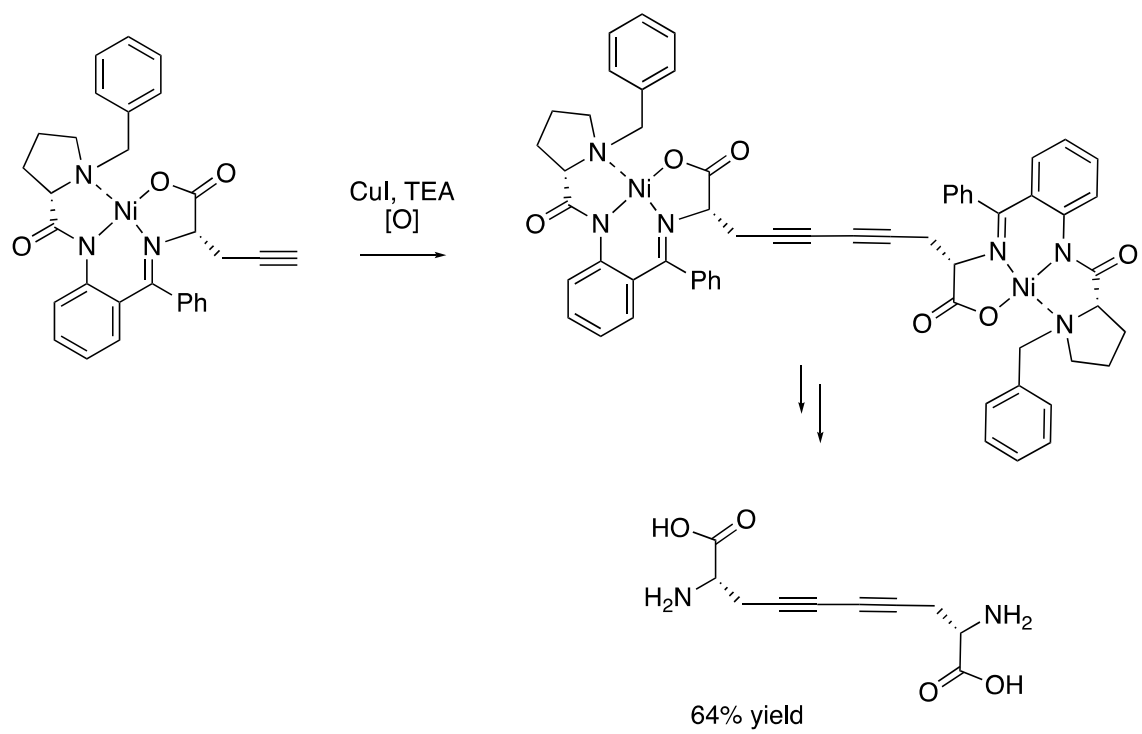

\section{Scheme 2}

\subsection{C-H Functionalization in Co-complexes}

A unique methodology for vinylic $\mathrm{C}-\mathrm{H}$ functionalization in unactivated alkenes exploiting ligand-based reactivity of $\mathrm{Co}$ and $\mathrm{Ru}$ complexes, was developed by Bergman and Toste. ${ }^{9}$ In situ trapping of the $\mathrm{CpCo}(\mathrm{I})(\mathrm{NO})_{2}$ reactive species with unactivated alkenes via an orbital symmetry-allowed ligand-centered [3+2] cycloaddition reaction afforded dinitrosoalkane complexes possessing relatively acidic $\mathrm{Csp}{ }^{3}-\mathrm{H}$ bonds amenable to deprotonation and functionalization with electrophilic acceptors (Scheme 3). The entire sequence starting from the Co or Ru complex precursors and the alkenes, that ultimately afforded products with vinylic carbons functionalized with the selected electrophiles, could be realized as a one-pot protocol stoichiometric in Co or Ru (Scheme 3). ${ }^{9}$

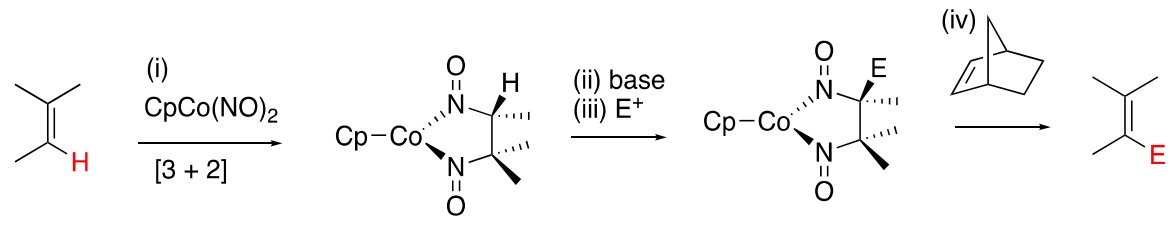

\section{Scheme 3}

Extensive studies on reactions of these stoichiometric nitrosyl complexes of Co and Ru culminated in the development of asymmetric synthesis of valuable chiral nonracemic bidentate ligands (Scheme 4). In the presence of chiral nonracemic strong base (NaHMDS with quaternary ammonium salt additive $\mathrm{R}^{*}{ }_{4} \mathrm{NCl}$ derived from cinchona alkaloids), desymmetrization of the Co scaffold via stereoselective deprotonation is followed by the preferential attack on one enantiotopic face of the prochiral enone to deliver the monofunctionalized CpCo(alkane) complex in excellent diastereoselectivity. ${ }^{10}$ Iterative application of this protocol to both the double bonds in the norbornadiene substrate was made possible by thermodynamically driven isomerization of the enantioenriched Co complexes produced in the first step. Overall, the iterative procedure afforded bisfunctionalized product strongly favoring anti-isomers over the syn-isomers, and delivering each as a single diastereomer that afforded alkene products in high enantiopurity after decomplexation (ee 90-96\%) prior to crystallization (Scheme 4). 


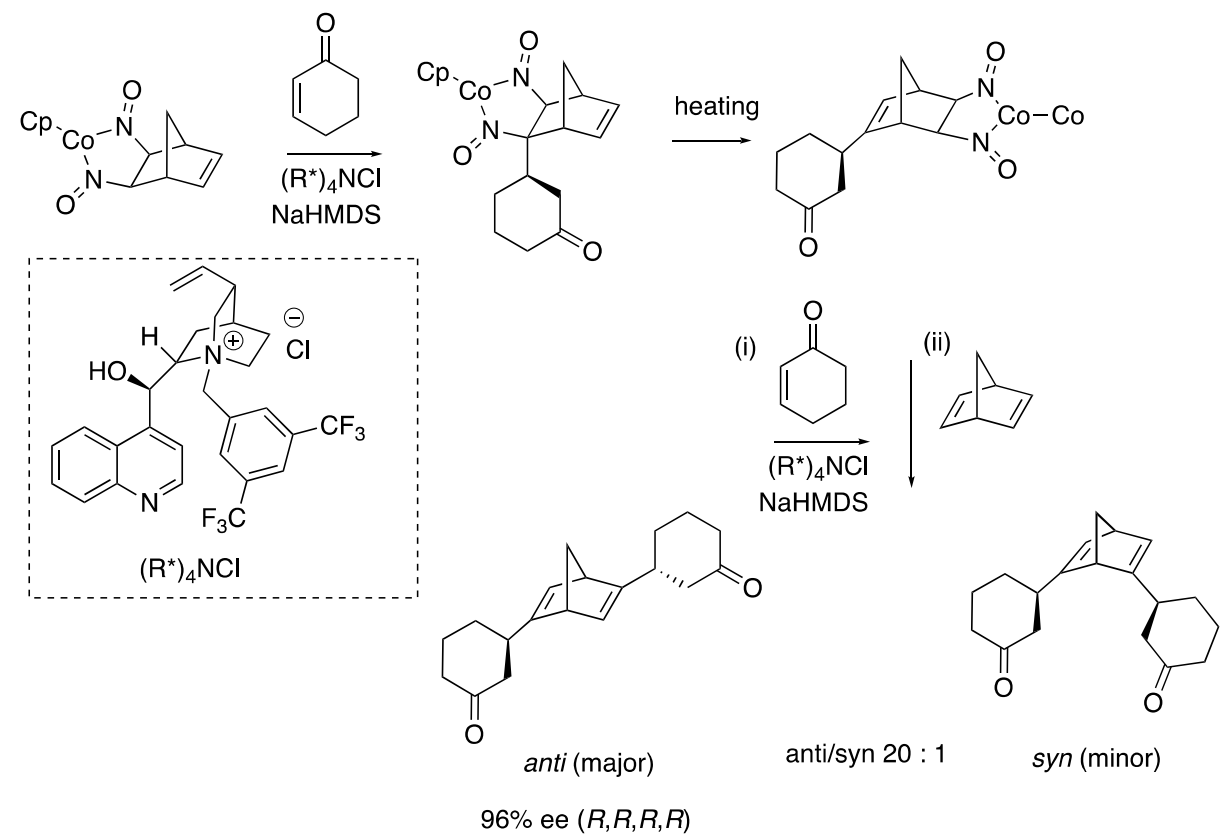

\section{Scheme 4}

Treatment of a racemic Co scaffold with the chiral nonracemic base followed by a diastereoselective intramolecular Michael reaction achieved an efficient relay of the stereochemical information to a new stereocenter at the $\beta$-position of the Michael acceptor. Decomplexation afforded the alkene in significant (57\%) enantiomeric excess (Scheme 5). ${ }^{9,10}$

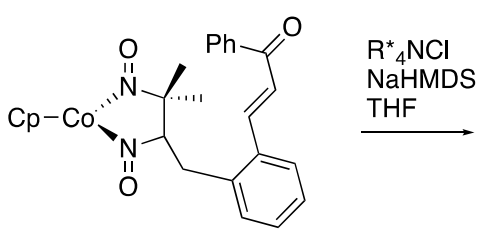

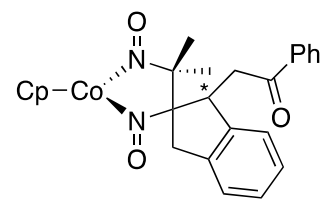

$57 \%$ yield
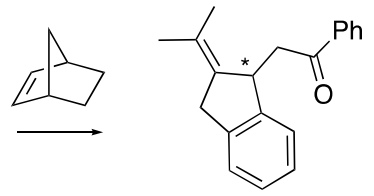

$95 \%$ yield, $57 \%$ ee

\section{Scheme 5}

However, although Co-catalyzed vinylic functionalization was demonstrated in principle in $2011,{ }^{11}$ the limitations in the scope of the catalytic process have not been overcome to date (Scheme 6).

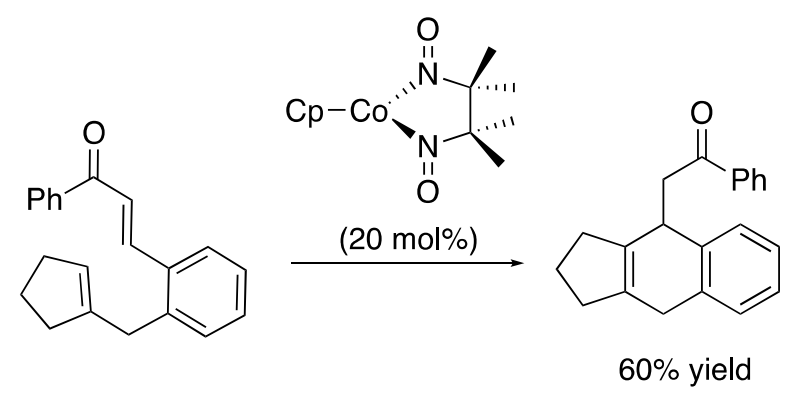

\section{Scheme 6}




\subsection{C-H Functionalization in $\mathrm{Cr}$-arene complexes}

The $\eta^{6}$-aryl chromium tricarbonyl complexes pioneered in the past by Semmelhack and others ${ }^{5,6}$ as scaffolds for functionalization of aromatic rings (vide infra), have recently been applied as catalysts in asymmetric synthesis. ${ }^{12}$ Consequently, various methodologies for stereoselective elaboration of $\eta^{6}$-aryl chromium tricarbonyl complexes have been actively sought in recent years.

Deprotonation of a chiral nonracemic $\eta^{6}$-arene chromium tricarbonyl complex featuring a chiral auxiliary group in the arene ligand followed by reaction with an electrophile afforded the corresponding 1,2disubstituted chromium arene complex with excellent diastereoselectivity (dr > $99: 1$ ) (Scheme 7). ${ }^{13}$ Analysis of the stereochemical outcomes for a series of reactions supported a mechanism involving selective coordination of the lithium ion of the base to the nitrogen heteroatom in the arene substituent.

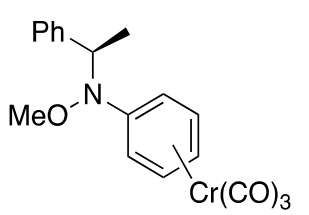

$(R)$

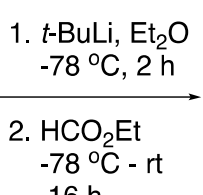

$16 \mathrm{~h}$

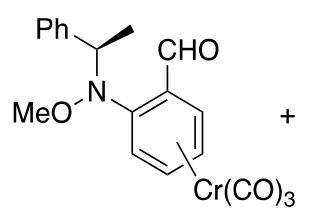

$82 \% \quad(S, R)$

$(\mathrm{dr}>99: 1)$

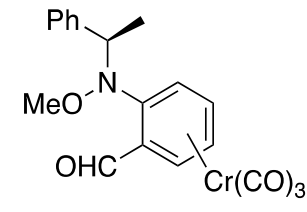

$4 \% \quad(R, R)$

\section{Scheme 7}

An approach to preparation of high enantiopurity $\eta^{6}$-arene chromium tricarbonyl complexes via a Pdcatalyzed asymmetric arylation has been reported (Scheme 8$).{ }^{14}$ The protocol utilizes functionalization of a benzylic $\mathrm{C}-\mathrm{H}$ bond activated for the reaction by the presence of the chromium metal center. ${ }^{14}$ The methodology was featured in a recent review on catalytic elaborations of $\eta^{6}$-coordinated arene transition metal complexes. ${ }^{15}$

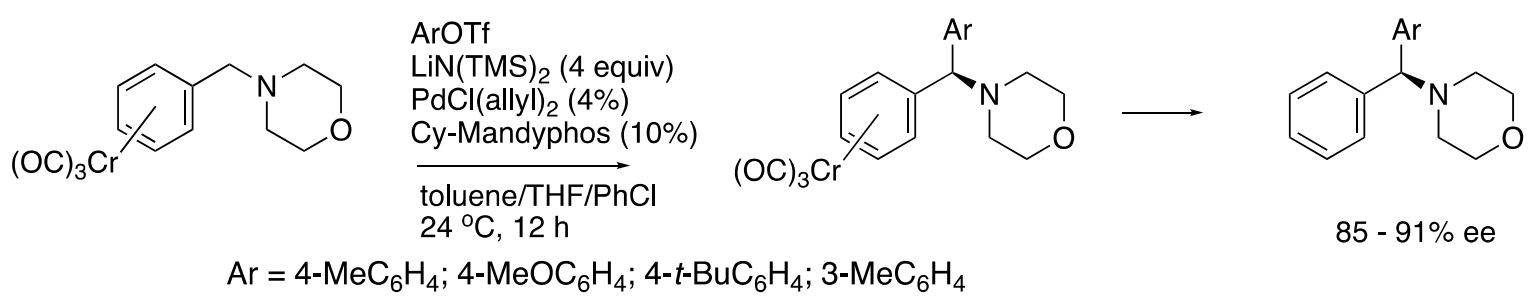

\section{Scheme 8}

\subsection{Complexes with a Pd-bonded stereogenic carbon}

At times, stable transition metal complexes are isolated fortuitously, as a result of a particular functionalization pattern in the ultimate synthetic target, and present opportunities for significant mechanistic investigations. In one such occurrence, during the asymmetric synthesis of a pyrrolidinoindoline alkaloid by Overman, a stoichiometric amount of $\mathrm{Pd}(\mathrm{OAc})_{2}$ was used to induce an intramolecular Heck reaction in the presence of a strong base ( $P M P=1,2,2,6,6$-pentamethylpiperidine) and a chiral nonracemic bidentate phosphine ligand. ${ }^{16}$ Unexpectedly, a stable six-membered palladacycle (67-95\% yield, $93: 7$ - $96: 4$ dr) notable for possessing a metal-bonded $\mathrm{sp}^{3}$-hybridized stereogenic carbon, as well as available $\beta$-hydrogens, was isolated. Treatment of the palladacycle with a weak acid (hydrotriflate of 2,6-di-tert-butylpyridine, TBP-HOTf) 
then afforded the targeted product of the intramolecular Heck reaction possessing a quaternary spirocenter, in 48\% yield and 95 : 5 er (Scheme 9).

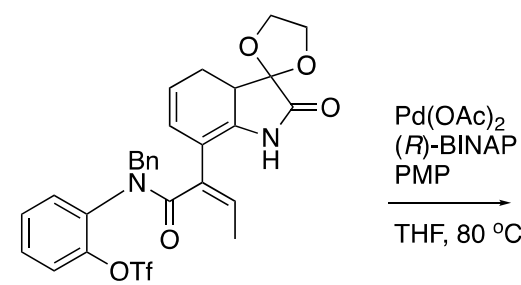

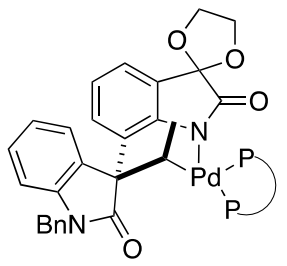

$\beta$-diastereomer

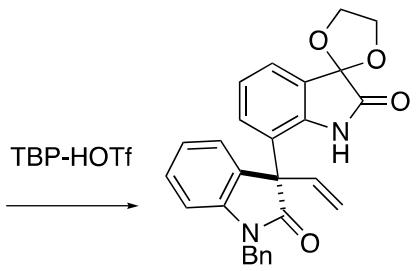

TBP = 2,6-di-tert-butylpyridine

(R)-BINAP: 95\% yield; dr $96: 4$

$(R, R)$-BDPP: $84 \%$ yield; dr $93: 7$

\section{Scheme 9}

In a subsequent study, the mechanism of epimerization of the palladium bonded stereocenter in the isolated palladacycles was investigated in more detail (Scheme 10). ${ }^{17}$ Palladacycles bearing $(R)$-BINAP and $(R, R)$-BDPP ligands were characterized by NMR spectroscopic methods, and in two cases by $\mathrm{X}$-ray crystallographic analyses. The epimerization took place under thermal conditions (above $120{ }^{\circ} \mathrm{C}$ ), or at room temperature in the presence of weak acids. A rapid equilibration of the originally formed $\beta$-diastereomer of the palladacycle to three cationic palladium complexes took place, slowly leading to the $\alpha$-epimer (Scheme 10). The process was rationalized as a sequence of protonation at the indoline nitrogen that opened the coordination site necessary for subsequent $\beta$-hydride elimination, and alkene re-insertion into the $\mathrm{H}-\mathrm{Pd}$ bond. Depending on the regiochemistry of the re-insertion, either the $\alpha$-diastereomer of the original palladacycle, or a cationic complex with a primary palladium-bonded carbon were obtained (Scheme 10).

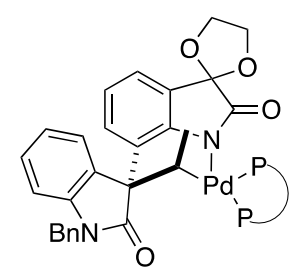

beta-diastereomer<smiles>C=CC=C</smiles>

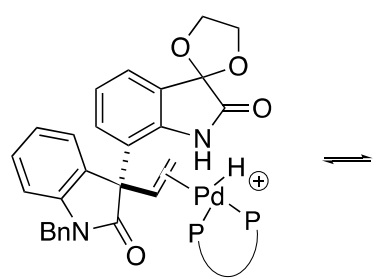

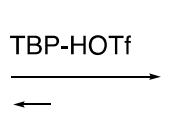

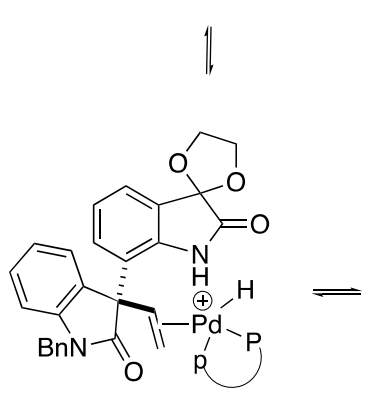

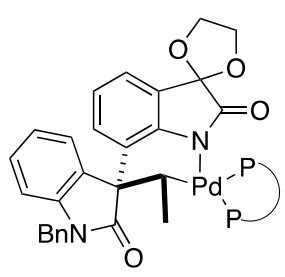

most stable

alpha-diastereomer

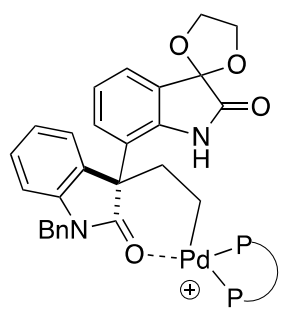


Unfortunately, the palladium-bonded stereocenter could not be further functionalized. Carbonylation of the $\beta$-diastereomer of the palladacycle in methanol under mild acidic conditions afforded exclusively a product with the methyl ester bonded to the primary carbon (Scheme 11).
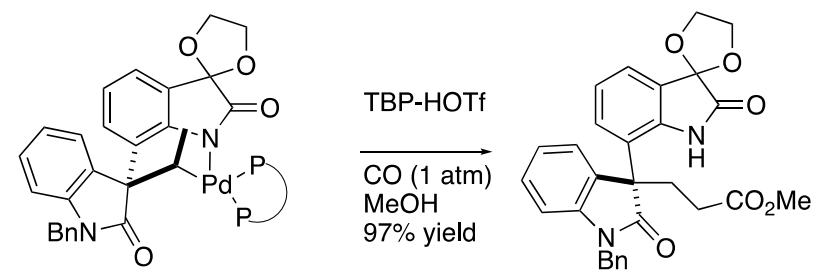

\section{Scheme 11}

Seeking to advance understanding of structure and reactivity of related complexes with palladiumbonded stereogenic carbons, stable palladacycles were designed by Malinakova. ${ }^{18}$ High enantiopurity stable five-membered palladacycles were prepared taking advantage of efficient stereoinduction from chiral nonracemic bindentate ligands $(1 R, 2 R)-N, N, N^{\prime}, N^{\prime}$-tetramethyl-1,2-diaminocyclohexane), and were characterized by X-ray crystallography. The ligand sphere created a chiral environment that effectively differentiated the enantiotopic faces of ester and amide enolates, giving rise to palladacycles with a metalbonded stereocenter in a significant diastereomeric excess (Scheme 12).

A highly diastereoselective ring closure of organopalladium complexes bearing the sulfonamide group (TfN) was realized following the enrichment of the palladium(II) iodide in one atropoisomer (arising from rotation about the Ar-Pd bond) to $98: 2$ ratio via pre-treatment with silica. Base-mediated ring closure under kinetic conditions ( $t$-BuOK, AgOAc, $-78^{\circ} \mathrm{C}, 10 \mathrm{~min}$ in THF) delivered the corresponding palladacycle in $\mathrm{dr}=96: 4$ ( $92 \%$ de), and essentially quantitative yield. In contrast, a palladium(II) iodide complex featuring the ether group could only be enriched to the ratio of atropoisomers $60: 40$, and afforded the corresponding palladacycle in $\mathrm{dr}=88: 12(64 \% \mathrm{de})$ by the base-mediated ring closure under thermodynamic conditions ( $t$ BuOK, rt, $60{ }^{\circ} \mathrm{C}$ in THF). The treatment of diastereomerically enriched palladacycles bearing a sulfonamide group with base in protic solvent $(t-\mathrm{BuOK} / t-\mathrm{BuOH}, \mathrm{rt}, 3 \mathrm{~h})$ resulted in significant erosion of the diastereomeric ratio (from $92 \%$ de to $57 \%$ de) due to epimerization of the Pd-bonded stereocenter. In contrast, treatment of the ether-derived palladacycles under the same conditions in fact achieved a slight diastereomeric enrichment (64\% to $74 \%$ de), reflecting the kinetic vs thermodynamic conditions for the original ring-closure. The palladium-bonded stereocenter proved to be configurationally stable during ligand-exchange with stabilizing bidentate phosphine ligands. The ligand exchange process yielded chiral nonracemic palladacycles featuring the palladium-bonded $\mathrm{sp}^{3}$-hybridized stereocenter as the only source of asymmetry, in enantiomeric excess (60\% ee), equal to the diastereomeric excess ( $60 \%$ de) of the substrate (Scheme 13$)$. 

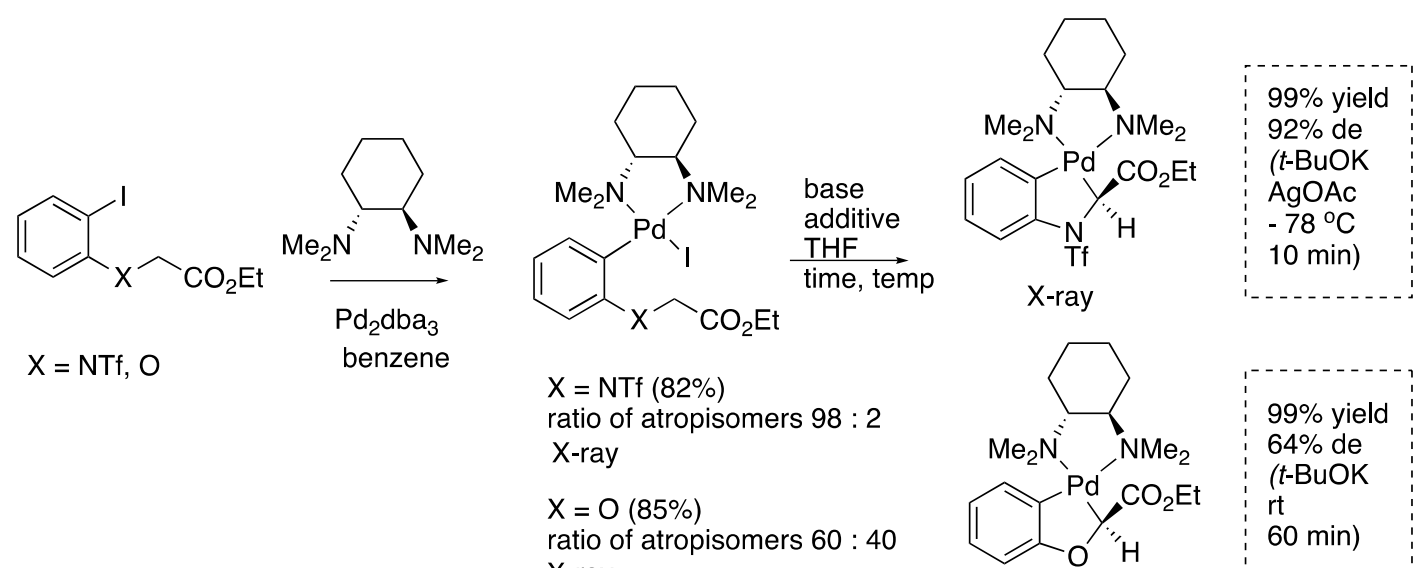

$$
\begin{aligned}
& X=N T f(82 \%) \\
& \text { ratio of atropisomers } 98: 2 \\
& X \text {-ray } \\
& X=O(85 \%) \\
& \text { ratio of atropisomers } 60: 40 \\
& \text { X-ray }
\end{aligned}
$$

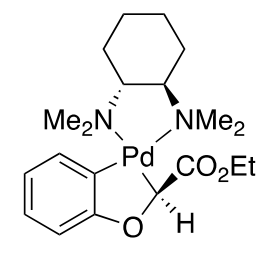

\section{Scheme 12}

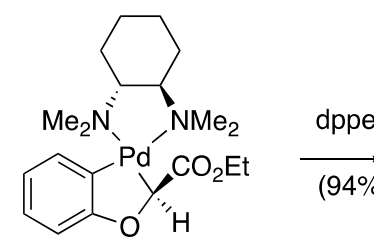

$60 \%$ de by ${ }^{1} \mathrm{H}$ NMR

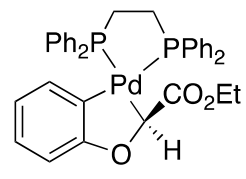

$60 \%$ ee by HPLC

\section{Scheme 13}

These chiral nonracemic palladacycles have been used to assess configurational stability of the palladium-bonded stereocenter in the absence of any chiral ligands (Scheme 14). ${ }^{18}$ The displacement of chiral 1,2-diaminocyclohexane ligands with achiral triphenyl phosphine afforded the corresponding nonracemic stable palladacycles, that were reacted with a series of alkynes to afford 1,2-dihydroquinolines and
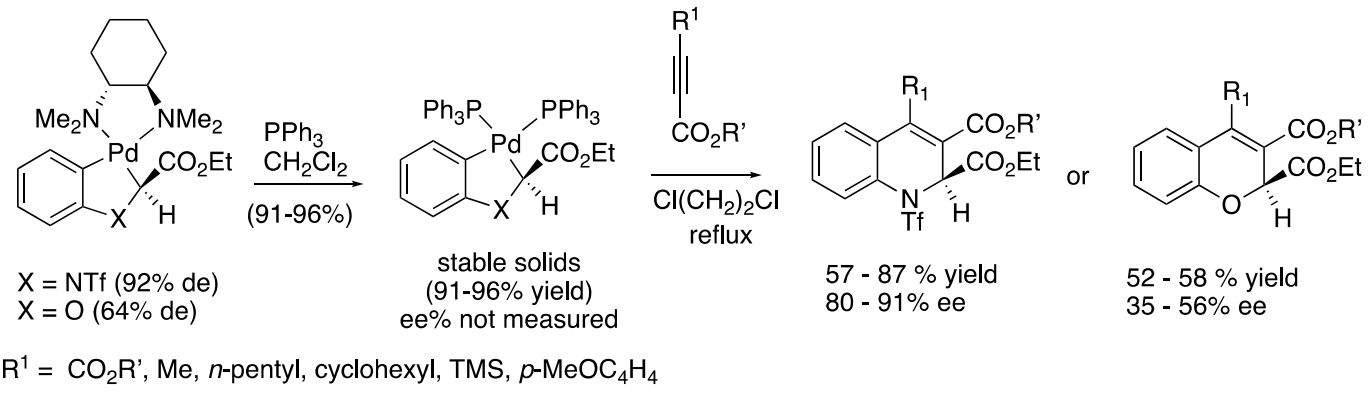

\section{Scheme 14}

$2 \mathrm{H}$-1-benzopyrans with retention of configuration to a significant extent at the palladium-bonded stereogenic carbon. Palladacycles bearing the sulfonamide functionality (assumed to have 92\% ee) showed high configurational stability of the metal-bonded stereocenter to afford a series of 1,2-dihydroquinolines in 80$91 \%$ ee pointing to a minimal racemization (6.5-13\%). In contrast, the palladacycles bearing the ether group 
(assumed to have 64\% ee) were more prone to racemization of the palladium-bonded stereocenter affording the $2 \mathrm{H}$-1-benzopyrans in $32-56 \%$ ee, revealing $37-50 \%$ erosion of enantiomeric purity.

The studies discussed above demonstrate the capability of a transition metal centre with its auxiliary ligand sphere to activate specific bonds in the organic fragment for further functionalization, as well as to control the stereochemical outcome of the process. The cited contributions applied these concepts to the development of new practically applicable synthetic methodologies, or for elucidation of fundamental pathways and properties of stable analogs of intermediates in catalytic transformations.

\section{Methods for Elaboration of Organometallic Complexes with Planar Chirality}

Functionalization of diverse polyhapto $\left(\eta^{2}-\eta^{6}\right)$-bonded organic ligands in stable transition metal (Os, Re, Fe, $\mathrm{Ru}, \mathrm{Cr}, \mathrm{Mo}$ ) complexes possessing planar chirality ${ }^{19}$ provided a foundation for the development of highly regioand stereocontrolled methods for synthesis of complex organic substrates. In order for these protocols to be become relevant tools for the $21^{\text {st }}$ century synthetic organic chemistry, the methods must be compatible with substrates featuring heteroatoms, as well as multiple sensitive functional groups. Furthermore, the contributions of the transition metal atom must be maximized, by exploiting the metal-induced reactivity modulation and stereocontrol in multiple sequential synthetic operations. Systems that allow for efficient control of absolute stereochemistry are the expected standard. The potential of these methodologies to allow for a highly modular, rapid and automated preparation of libraries of diverse organic products makes these synthetic approaches highly attractive to the pharmaceutical industry.

Undeniably, studies reported in this area over the past fifteen years met these standards and demonstrated the value of chiral nonracemic stoichiometric transition metal-based templates with planar chirality to modern synthetic chemistry.

\subsection{Elaboration of $\mathrm{Cr}$-, Fe- and Ru-complexes with planar chirality}

Nucleophilic addition and substitution reactions of $\eta^{6}$-coordinated arene ligands in cationic $\mathrm{CpRu}^{+}$or $\mathrm{CpFe}^{+}$ complexes and in neutral chromium tricarbonyl complexes were extensively studied in 1980s and 1990s, and were developed into valuable, albeit specialized, synthetic tools introducing reactivity patterns complementary to the classical chemistry of aromatic rings. ${ }^{19}$ However, the adaptation of these methodologies to asymmetric synthesis has presented a challenge.

Kundig ${ }^{20}$ achieved success utilizing stoichiometric chiral additives, or chiral directing groups in his asymmetric synthesis of both enantiomers of the natural product acetoxytubipofuran exploiting $\mathrm{Cr}(\mathrm{CO})_{3^{-}}$ mediated dearomatization (Scheme 15).

Pigge $^{21}$ applied dearomatization of arene ruthenium complexes constructed from enantiopure $(S)-\alpha$ methyl benzylamine ( $>98 \%$ ee) for a diastereoselective preparation of a single enantiomer of chiral nonracemic functionalized spirolactams (Scheme 16). 


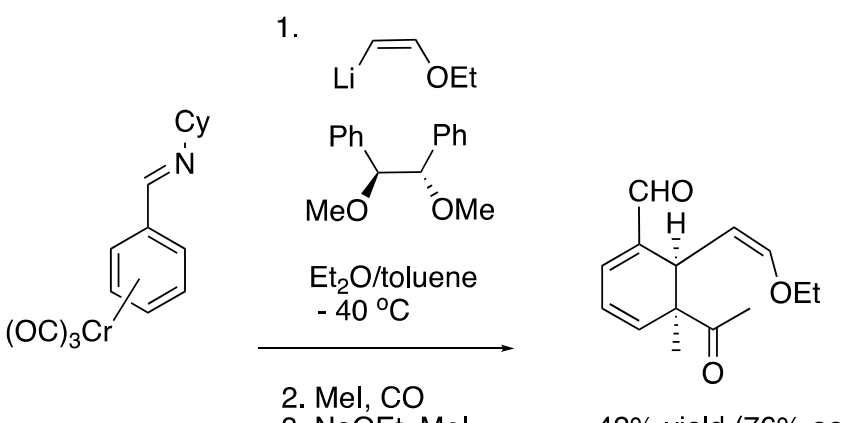<smiles>COC[C@H](/N=C/c1cccc(Cl)c1)C(C)C</smiles>

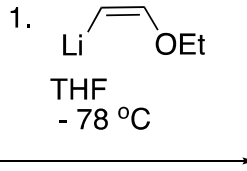

2. $\mathrm{Mel}, \mathrm{CO}$

3. NaOEt, Mel
$\mathrm{AcO}$<smiles>CC(C)=COCC1=CC=C[C@]2(C)Cc3occ(C)c3C[C@H]12</smiles>

acetoxytubipofuran<smiles>CCO/C=C\C1C(C=O)=CC=C[C@]1(C)C(C)=O</smiles>

$53 \%$ yield (>95\% ee)

\section{Scheme 15}

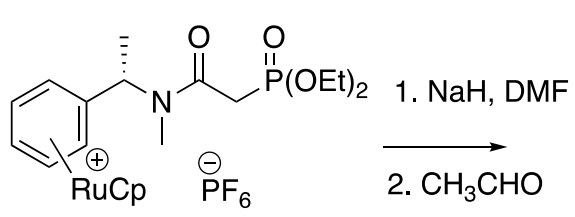

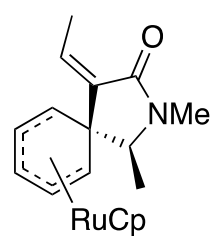<smiles>C/C=C1/C(=O)N(C)C2(C=CC=CC2O)C1C(C)OC(F)F</smiles>

$\mathrm{CpRu}(\mathrm{CO})_{2} \mathrm{Br}$ recovered

\section{Scheme 16}

Studies by Pearson, ${ }^{22}$ Knöelke, $^{23}$ and Stephenson ${ }^{24}$ developed iron cyclohexadienyl complexes into valuable synthetic templates with planar chirality, suitable for iterative 1,1- and 1,2-bisfunctionalization, thus opening new pathways for complex natural product syntheses (Schemes 17 and 18). Racemic $\eta^{4}$ cyclohexadienyl iron complexes were activated by oxidation to $\eta^{5}$-cationic iron complexes that acted as electrophiles poised for nucleophilic attack proceeding with exquisite selectivity from the diastereotopic face opposite to the iron center. Oxidative demetallation afforded dienes amenable to further diversification via Diels-Alder reactions (Scheme 17). ${ }^{23,24} \mathrm{~A}$ unique reaction pathway rationalized as diastereoselective [6+2] ene reaction was observed by Pearson (Scheme 17)..$^{22}$ 
H.-J. Knöelke (2004)

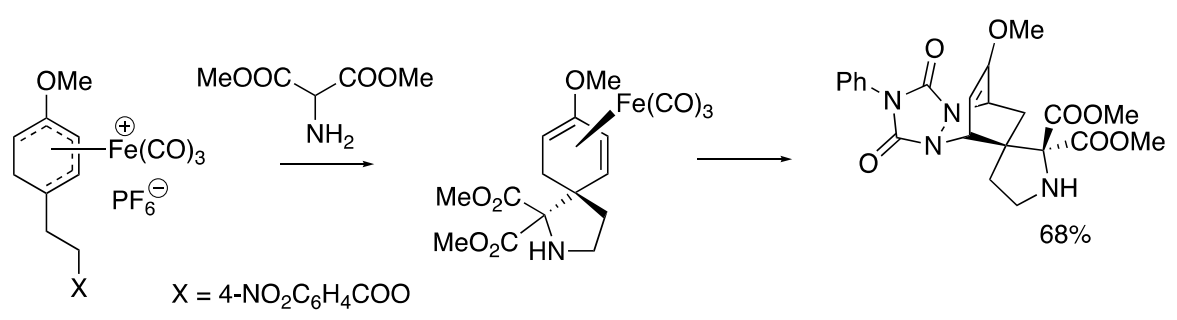

A. J. Pearson (2007)
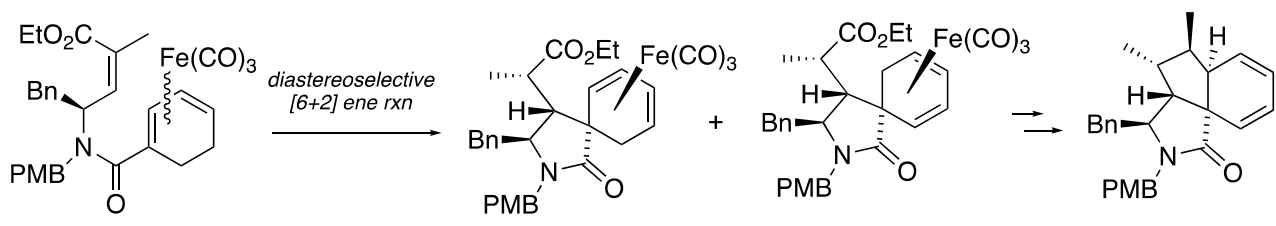

\section{Scheme 17}

Particularly notable are methodologies designed by Stephenson ${ }^{25,26}$ for the installation of the quaternary carbon stereocenter in applications to racemic syntheses of Amaryllidaceae alkaloids (Scheme 18).

G. R. Stephenson (2011)
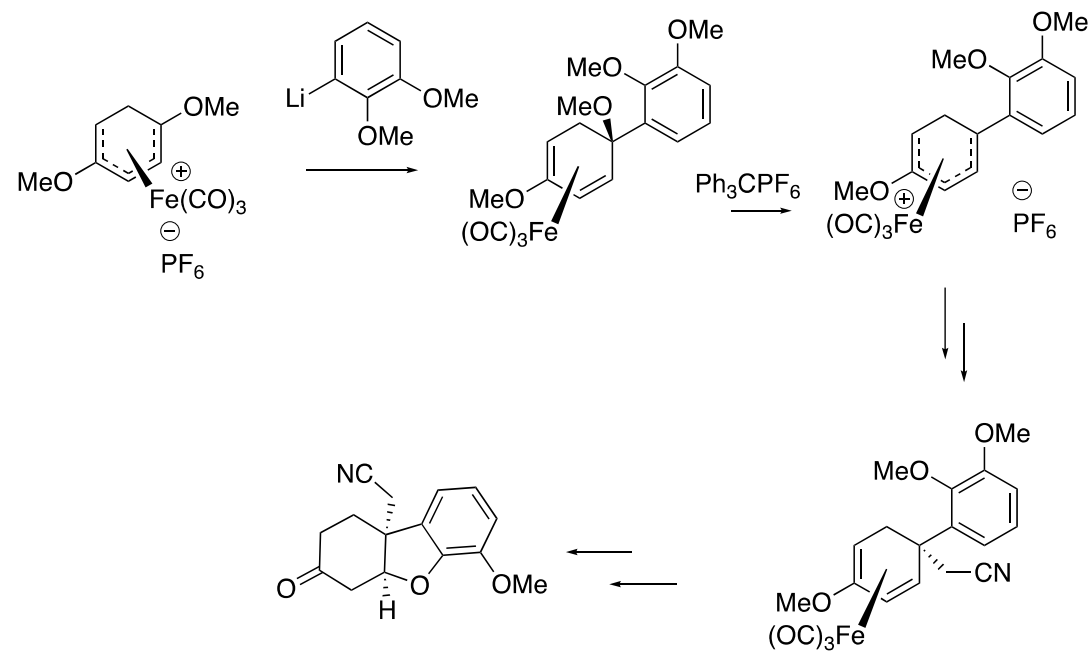

G. R. Stephenson (2008)

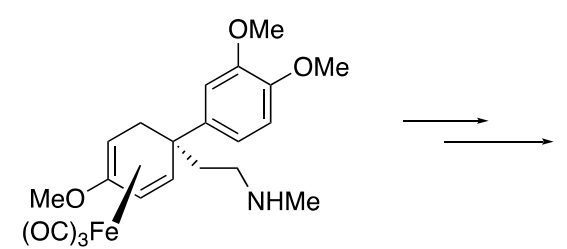<smiles>COc1ccc([C@]23CCC(=O)C[C@H]2CN3C)cc1OC</smiles>

\section{Scheme 18}

One notable deficiency in the current state-of-the-art of iron cyclohexadienyl chemistry has been the lack of asymmetric transformations. Towards this goal, preparation of chiral nonracemic iron cyclohexadienyl complexes utilizing enantiopure 1,2-diols obtained by enzymatic dihydroxylation of aromatic substrates, has 
been recently achieved (Scheme 19). ${ }^{27}$ The protocol involved a preferential complexation of the iron syn to the positions of the free hydroxy groups on the cyclohexadiene substrate. However, when the activation of the iron cyclohexadienyl complex for the nucleophilic attack via acetate abstraction was attempted, the major pathway involved an achiral $\eta^{5}$-cationic iron intermediate, and therefore produced racemic products following the nucleophilic addition. Only small amounts of chiral nonracemic products were produced by the nucleophilic attack on the minor chiral nonracemic $\eta^{5}$-cationic iron complex. ${ }^{27}$ Nevertheless, impressive application to the synthesis of racemic oseltavimir was achieved by this methodology (Scheme 19). ${ }^{27}$
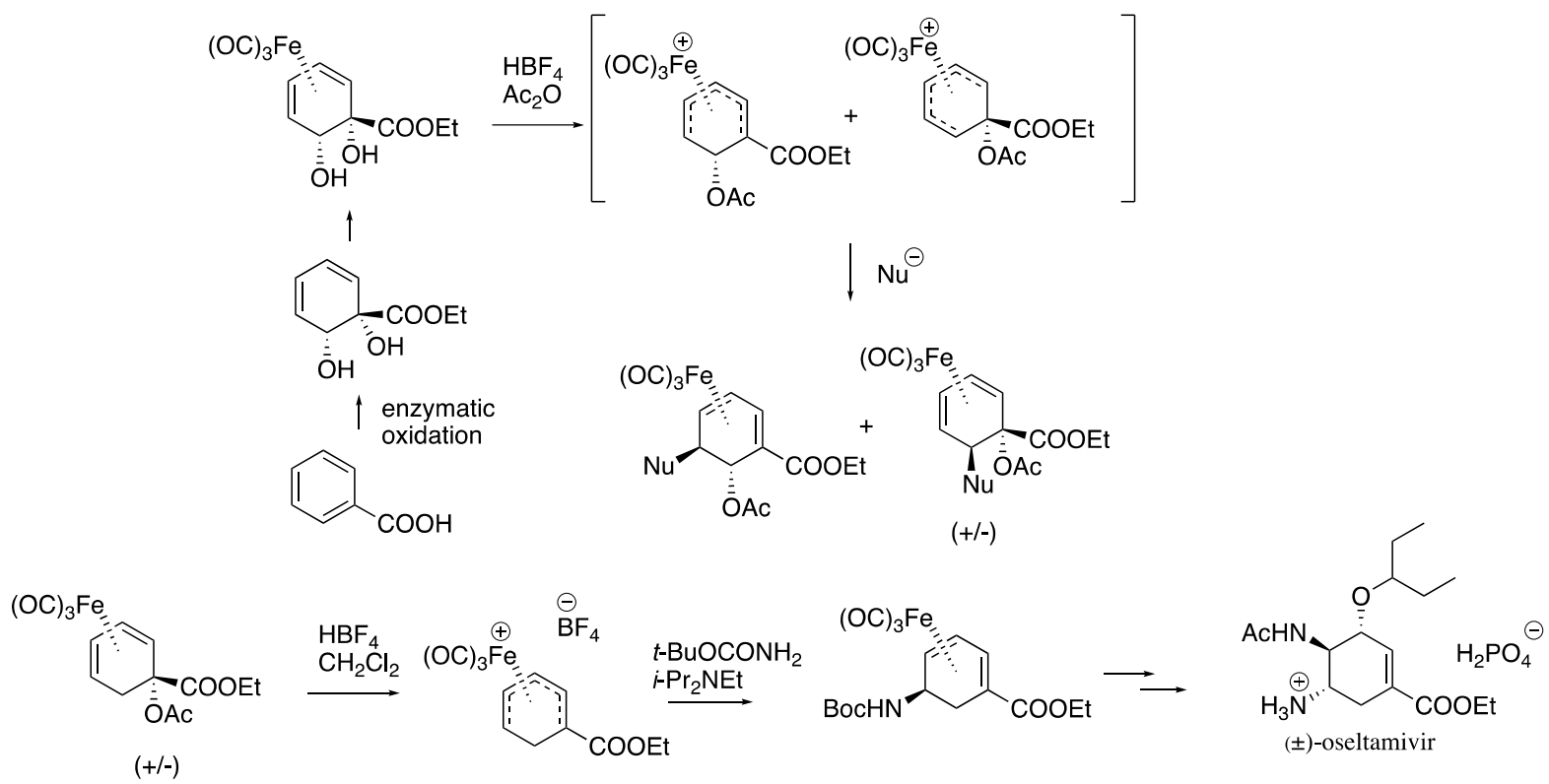

\section{Scheme 19}

A chiral nonracemic $\eta^{4}$-cyclohexadienyl iron complex prepared from the product of enzymatic dihydroxylation of sodium benzoate, was successfully applied in a concise biomimetic synthesis of (+)grandifloracin (Scheme 20). ${ }^{28}$ The synthesis features Diels-Alder dimerization of a chiral nonracemic cyclohexadienone in the ultimate step. The complexation of the chiral diene with the iron centre served as an effective protecting group strategy necessary to achieve the requisite functionalizations of the chiral diene, which would be rather sensitive to rearomatization in the absence of the iron coordination (Scheme 20).
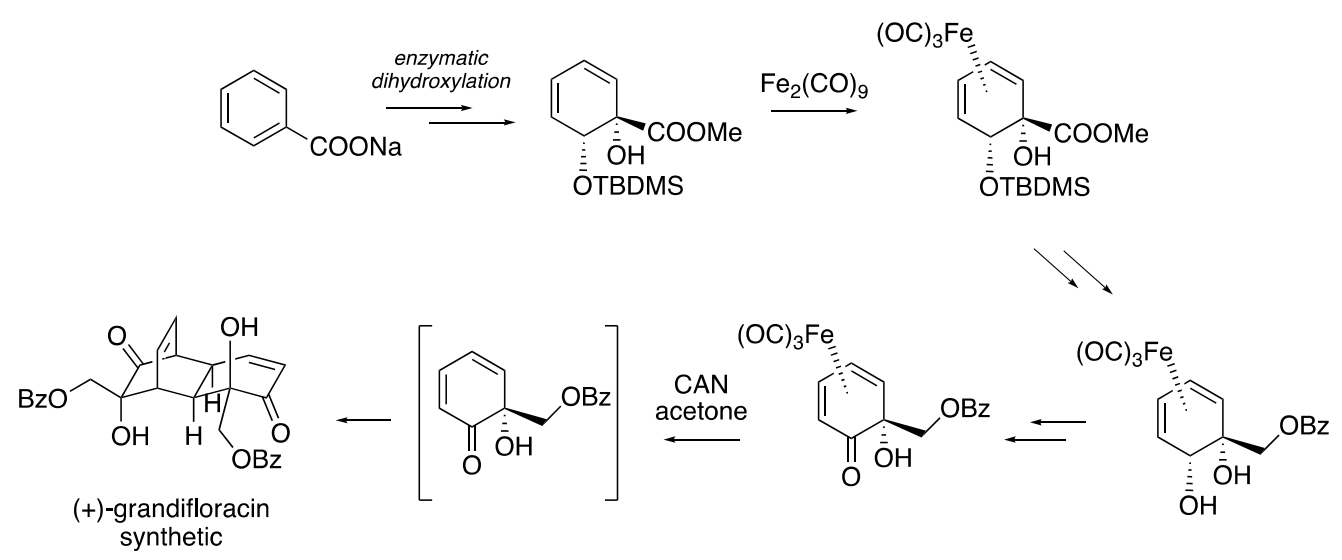

Scheme 20 


\subsection{Elaboration of Mo-Complexes with Planar Chirality}

A remarkably comprehensive methodology for the construction of high enantiopurity functionalized heterocycles has been developed by Liebeskind over 20 years of systematic exploration of reactivity nuances of stoichiometric molybdenum complexes of pyranyl and pyridinyl heterocycles, while keeping a keen eye for the needs and challenges of synthetic organic and medicinal chemistry.

The early work by Liebeskind ${ }^{29,30}$ firmly established the foundations of the methodology based on the preparation of neutral $\eta^{3}-3,4,5$ - or $\eta^{3}-2,3,4$-allylpyranyl or -piperidinyl complexes, as well as the $\eta^{3}-2,3,4$-allyl5,6-unsaturated variants, and 2,6-dimethoxy-substituted $\eta^{3}-3,4,5$-allyl analogs (Scheme 21), by processes based on those well known in classical molybdenum chemistry. ${ }^{31,32}$

Various approaches to the synthesis of enantiopure complexes were developed, including enzymatic kinetic resolution of acetate precursors, ${ }^{33}$ efficient separations of diastereomeric complexes bearing various chiral auxiliary groups on the organic fragments ${ }^{34,35}$ and a unique desymmetrization of meso complexes via diastereoselective OMe abstraction. ${ }^{36}$

Initial studies aimed at asymmetric synthesis of biologically relevant heterocycles culminated in the development of modular enantioselective procedures for 2,6-bisfunctionalization of the pyranyl and piperidinyl scaffold, relying on the regiocontrolled in situ generation of the $\mathrm{TpMo}(\mathrm{CO})_{2}$-stabilized cationic diene intermediate complexes (by hydride or $\mathrm{MeO}$ abstraction) that reacted with hard organic nucleophiles $(\mathrm{RM}, \mathrm{RMgBr})$ in a stereocontrolled manner attacking always opposite to the face bonded to the $\mathrm{TpMo}(\mathrm{CO})_{2}$ fragment. Several regioselective oxidative demetallation protocols were developed to generate reactive functionalities on carbons C3-C5 allowing for further elaboration of the heterocyclic products via alternative pathways, for example delivering selectively both diastereomers of 2,3,6 -trisubstituted piperidines, 2,3-cis3,6-cis vs 2,3-trans-3,6-trans, needed for the synthesis of the indolizidine alkaloid shown in Scheme $21 .{ }^{30} \mathrm{~A}$ few years later, an extensive study demonstrating the application of this methodology to medicinal chemistry disclosed the preparation of an extensive library of substituted piperidines, including five enantiomerically pure pyrrolizidine alkaloids, and detailed more recent advances and improvements in the preparation of the relevant molybdenum scaffolds on a large scale. ${ }^{37}$ 


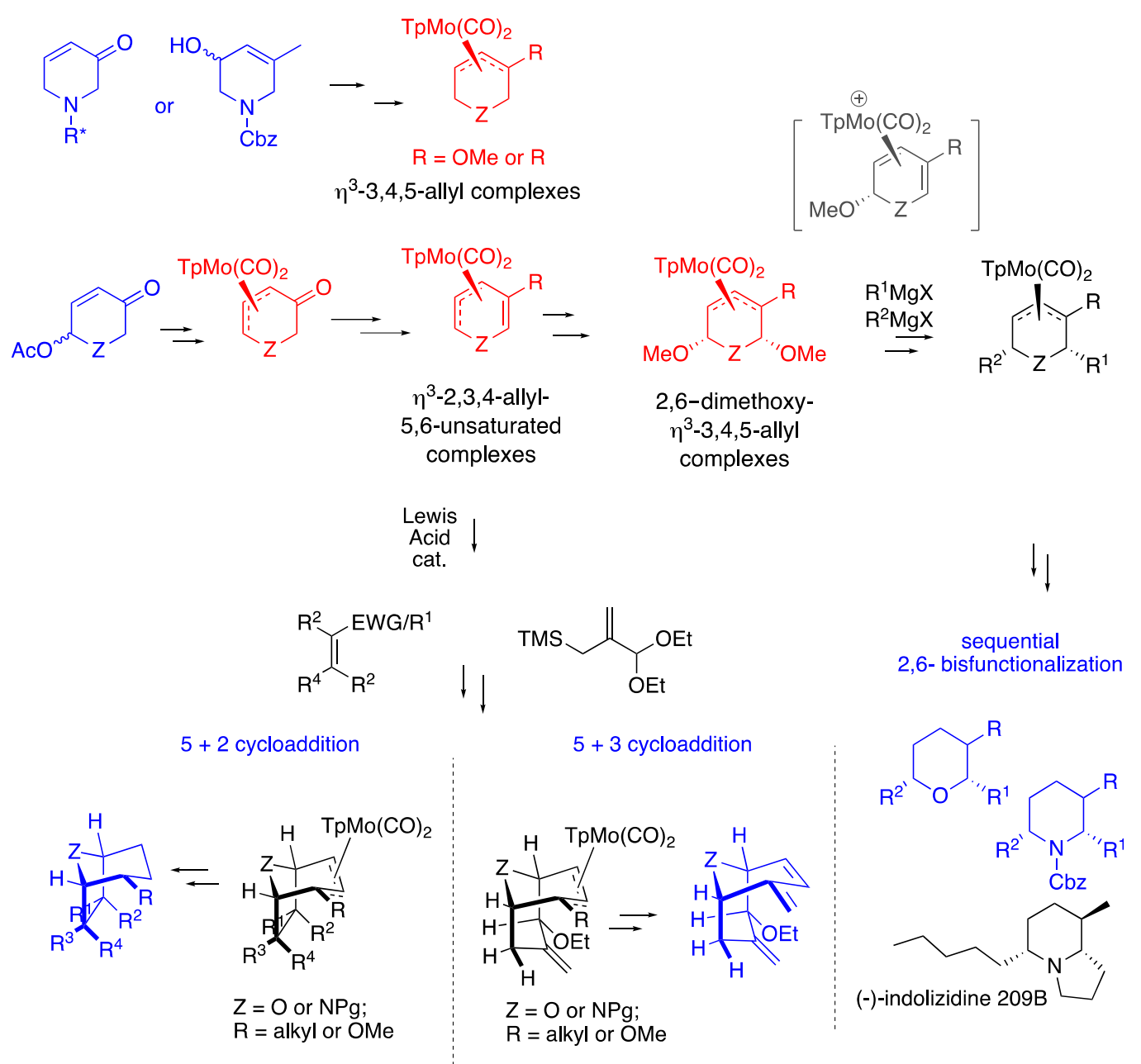

\section{Scheme 21}

In parallel, a powerful methodology based on [5+2], and a more limited [5+3] cycloaddition reaction, of the 5,6-unsaturated molybdenum pyranyl and piperidinyl complexes with Lewis acid-activated electrondeficient olefins was being developed (Scheme 21). ${ }^{38,39}$ In these protocols, the TpMo(CO $)_{2}$ fragment played its traditional role stabilizing the incipient positive charge, thus efficiently modulating the construction of [3.2.1] and [3.3.1] bridged heterocycles in high enantiomeric purity (Scheme 21). The cycloaddition methodology was also applied to the construction of fused annulated pyrans (Scheme 22). ${ }^{40}$ The electron-rich 1,3-diene activated by the presence of the $\mathrm{TpMo}(\mathrm{CO})_{2}$ scaffold was obtained by elaboration of the pyranyl complex prepared by methods shown in Scheme 21. The subsequent reactions with activated olefins under Lewis acid catalysis proceeded with excellent facial selectivity imposed by the bulky $\mathrm{TpMo}(\mathrm{CO})_{2}$ moiety. The [4+2] cycloaddition provided an unsaturated $\eta^{3}-2,3,4$-allyl molybdenum complex that afforded opportunities for two additional functionalization steps prior to demetallation. An intriguing switch in regioselectivity, likely due to a mechanistic divergence (via ene-like reaction), was observed for terminally substituted dienes (Scheme 22). 


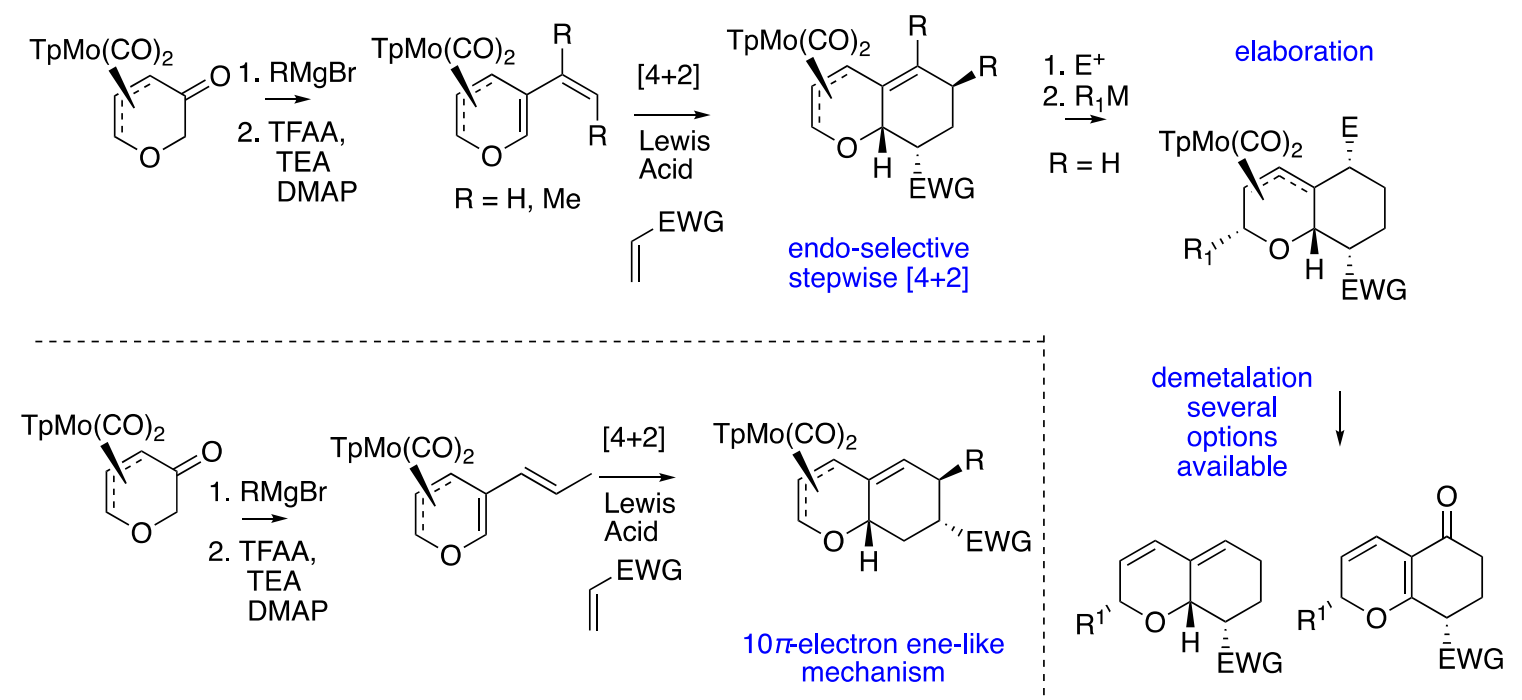

\section{Scheme 22}

These studies provide just a glimpse into the future possibilities of the "chiral transition-metal scaffolding" concept in rapid construction of biologically relevant $\mathrm{O}$ - and $\mathrm{N}$ - heterocycles via modular synthetic sequences. The advances reported by the Liebeskind group in recent years ${ }^{37,41-47}$ illustrate how a novel synthetic protocol can mature and continue to provide unexpected applications when a solid mechanistic understanding of every detail is sought in the early studies.

Notably improved, and consolidated preparations of chiral nonracemic TpMo(CO $)_{2}\left(\eta^{3}\right.$-oxopyranyl) and TpMo(CO) $)_{2}(\eta 3$-oxopiperidinyl) molybdenum scaffolds based on the oxa- and aza-Achmatowicz reaction, were reported. ${ }^{41}$ Complexes that serve as building blocks central to the entire Mo-scaffolding-based methodology, were obtained as stable compounds, in high enantiomeric purity and bulk quantities. Either enantiomer could be obtained with equal ease. Notably, the synthetic sequence can utilize substituted furans, thus allowing attachment of substituents at sites that were previously left unfunctionalized in the oxopiperidinyl and oxopyranyl complexes (Scheme 23).
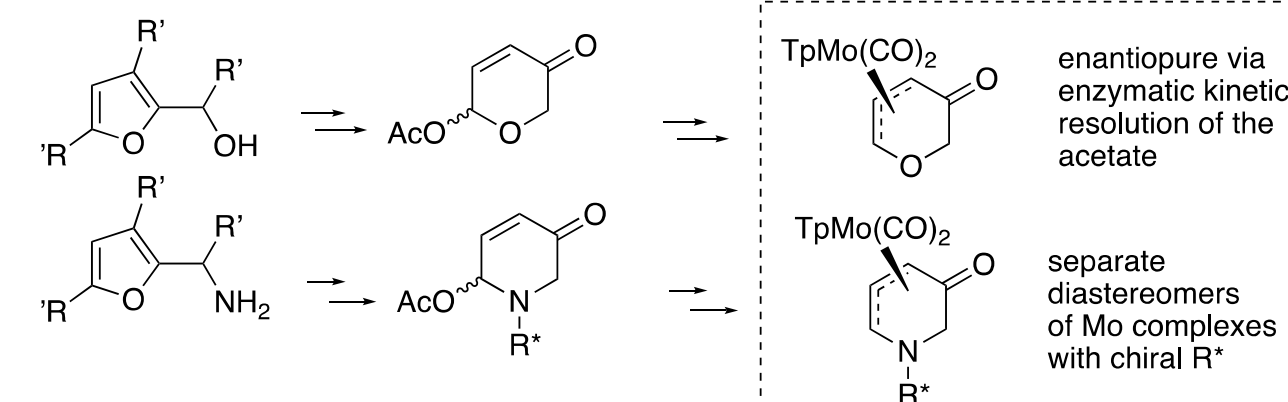

$$
\mathrm{R}^{\prime}=\mathrm{H}
$$$$
\mathrm{R}^{\star}
$$
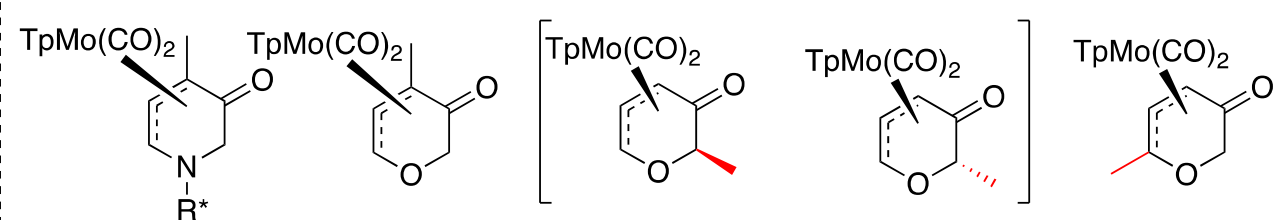

\section{Scheme 23}


An alternative synthetic protocol was developed for the construction of complementary unsaturated $\eta^{3}$-pyranyl and $\eta^{3}$-piperidinyl complexes, featuring a variable substituent at the C-6 (rather than C-5) position. ${ }^{42}$ Both molybdenum complexes were obtained in a high enantiomeric purity, utilizing distinct strategies (chiral pool vs resolution of diastereomeric aza complexes). The 5,6-unsaturated $\eta^{3}$-pyranyl and $\eta^{3}$ piperidinyl complexes reacted as anticipated in the [5+2] cycloaddition protocol, affording bridged heterocycles featuring quaternary all carbon stereocenters (Scheme 24). Completion of this missing element in the substitution pattern of the products will have a paramount impact on the value of this methodology to the construction of structurally diverse libraries of enantiopure heterocycles. Mechanistically, the study uncovered an unexpected requirement for the catalytic presence of a Bronsted acid, (AcOH) along with Lewis acid $($ EtAlCl 2$)$ for certain $R$ substituents $(R=P h) .{ }^{42}$

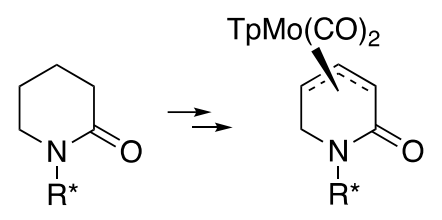

both enantiomers available in $>99 \%$ ee after resolution when using chiral auxiliary

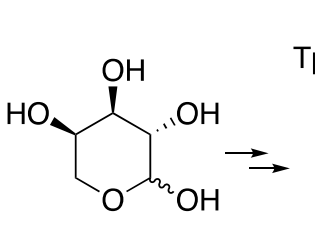

chiral pool approach to enantiopure scafold

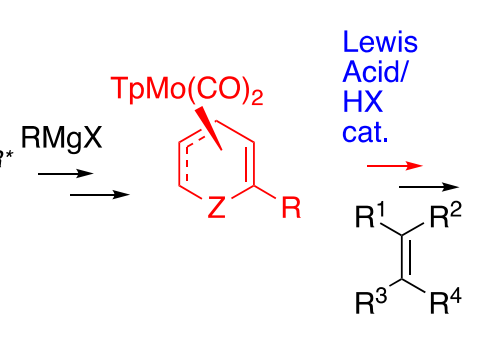

$5+2$ cycloaddition
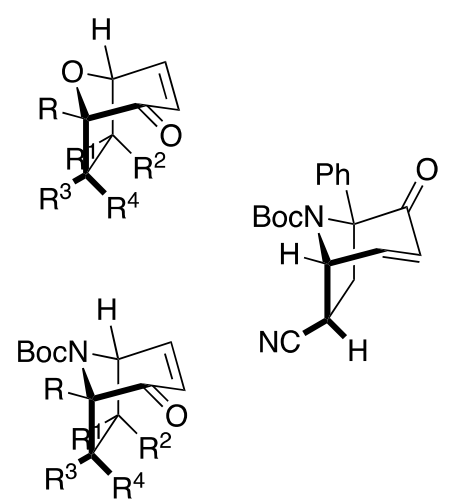

\section{Scheme 24}

In a series of several significant and extensive publications, the Liebeskind group has developed truly distinct reactivity patterns for the chiral nonracemic oxo-pyranyl and oxo-piperidinyl molybdenum scaffolds (Scheme 25). ${ }^{43-45}$ The protocols start with functionalization of the C- 6 carbon taking advantage of reactivities typical for the $\alpha$-carbon in carbonyl compounds. The transformations include: (i) the aldol reaction ${ }^{43,44}$ (ii) Mukaiyama-Michael reaction ${ }^{43,44}$ or (iii) a sequence of an aldol reaction, dehydration, attack of RMgX on a carbonyl group followed by semipinacol rearrangement. ${ }^{45}$ These steps are highlighted by blue-colored labels in Scheme 25.

The semipinacol rearrangement of the tertiary alcohol afforded another unique molybdenum scaffold with a quaternary carbon at the C-6 position, that proved applicable to asymmetric synthesis of alkaloid (-)adaline. ${ }^{45}$ In the semipinacol rearrangement, the additional stabilization of the adjacent positive charge by the Mo-centre is likely reinforcing the expected outcome. It is notable that such traditional reactivity patterns for organic functionalities occur smoothly on the organometallic scaffolds, and the $\mathrm{TpMo}(\mathrm{CO})_{2}$ fragment serves efficiently as a powerful stereo controlling element, directing the new bond formation to the face opposite to the metal centre. ${ }^{45}$

In the subsequent steps of the protocols summarized in Scheme 25, unprecedented transformations of the neutral $\eta^{3}$-allylmolybdenum complexes were observed, and are highlighted by red-colored labels. Remarkably, the stable neutral $\eta^{3}$-allylmolybdenum complex underwent an intramolecular nucleophilic attack by either the enolate or the alkoxide giving rise to unstable anionic $\eta^{2}$-bonded six-coordinated Mo complexes. 
These anionic complexes could be either trapped with methylating agents $\left(\mathrm{Me}_{3} \mathrm{OBF}_{4}\right)$ to stabilize the repositioned $\eta^{3}$-allylmolybdenum complex or subjected to in situ oxidative demetallation, as is shown in the synthesis of a derivative of natural brevicomine (Scheme 25). ${ }^{43}$ This is a significant observation in terms of fundamental reactivity of $\eta^{3}$-allylmolybdenum complexes, that also opens up an access to a much greater variety of complex organic structures. This point has been illustrated by completing two natural product syntheses (2-hydroxy-exo-brevicomin ${ }^{36}$ and (-)-adaline) (Scheme 25). ${ }^{43-45}$ The new reactivity of the molybdenum complex was rationalized by the preference for six-coordinate over seven-coordinate systems, and the availability of highly $\pi$-back-bonding ligands (two CO ligands and the $\eta^{2}$-enone) capable of stabilizing the negative charge on molybdenum.

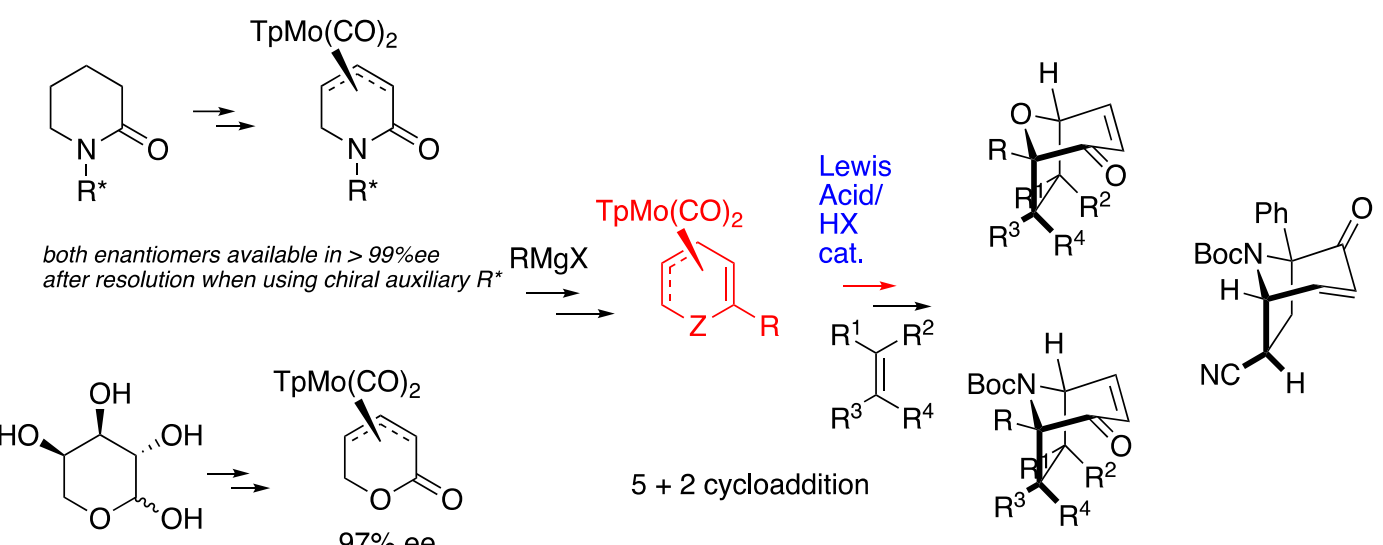

chiral pool approach to enantiopure scafold

\section{Scheme 25}

An unprecedented reactivity has also been reported for $\eta^{3}$-allylmolybdenum complexes featuring an excellent leaving group (acetoxy or trifluoroacetoxy) in the C-5 position of the heterocyclic substrate. ${ }^{46,47}$ Thus, 5-acetoxypyranyl and 5-acetoxypiperidinyl $\mathrm{TpMo}(\mathrm{CO})_{2}$ scaffolds reacted with stabilized carbon nucleophiles, as well as with electron-rich olefins, via an intermolecular $\mathrm{S}_{\mathrm{N}} 2^{\prime}$-like nucleophilic substitution to afford richly functionalized molybdenum complexes (Scheme 26). The value of a strategic deployment of the various transformations available to the molybdenum scaffolds has been demonstrated by a step-wise sequential $\mathrm{S}_{\mathrm{N}} 2$ 'nucleophilic attack/oxidative demetallation protocols that afforded various medicinally relevant molecules and/or natural products in a high enantiomeric purity (Scheme 26). It is important to point out that a Mostabilized carbocation was not generated as an intermediate in the $\mathrm{S}_{\mathrm{N}} 2^{\prime}$-like reactions. Control experiments performed with the cationic intermediate generated by alternative methods did not indicate the same reactivity patterns with stabilized nucleophiles. ${ }^{47}$ 


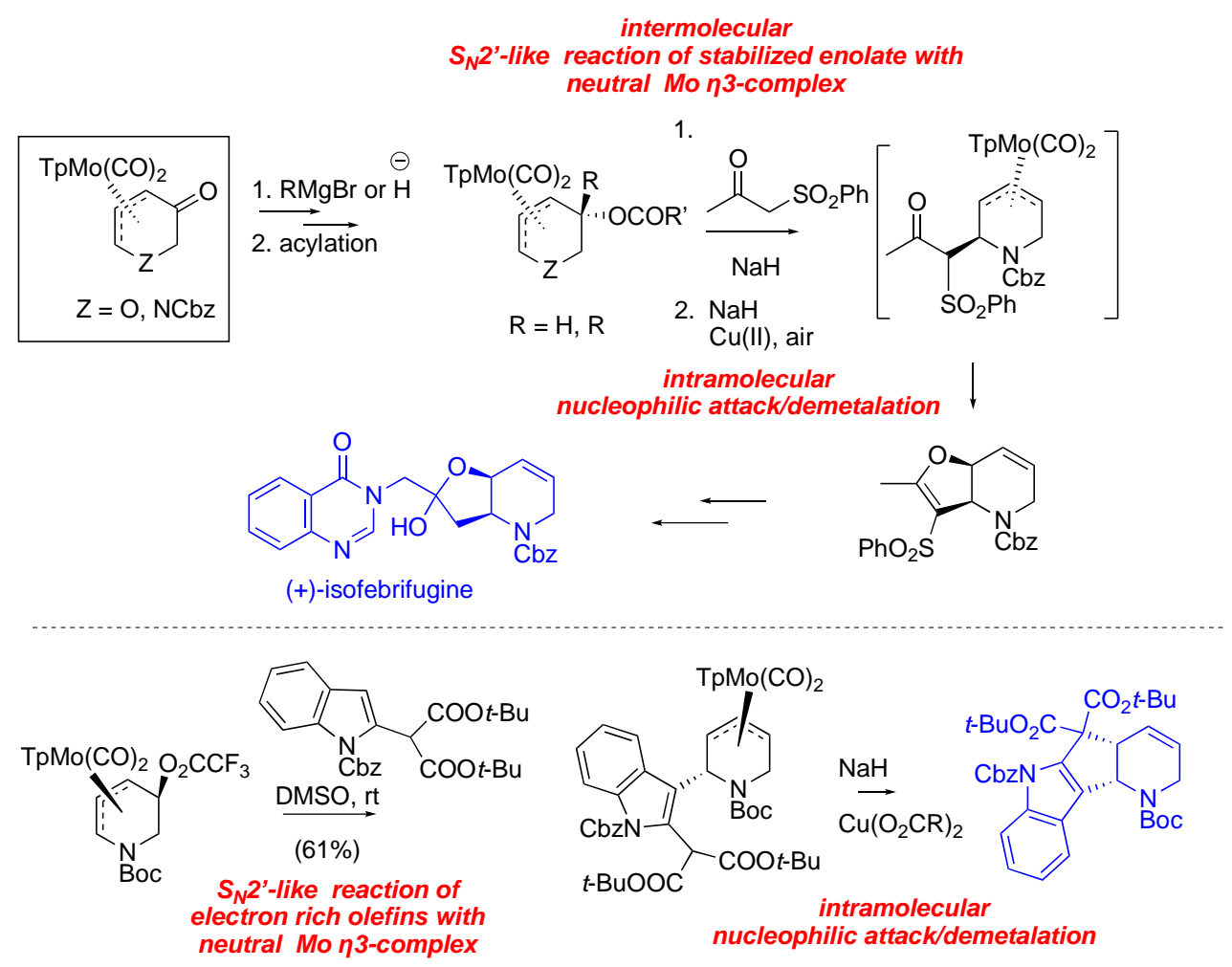

\section{Scheme 26}

\subsection{Dearomatization via $\eta \mathbf{2}$-Coordination with $\mathrm{W}$ and Mo}

Since early 2000s Harman's group has been developing a synthetic avenue for asymmetric synthesis that applies transition metal complexes of $\mathrm{Os}, \mathrm{Re}, \mathrm{W}$ and Mo as tools for desymmetrization of inexpensive, widely available aromatic and heteroaromatic substrates via selective $\eta^{2}$-coordination. In contrast to the traditional $\eta^{6}$-coordinated complexes between aromatics and $\mathrm{Cr}, \mathrm{Mn}$, Fe, Ru that activate the organic ligand for the attack by the nucleophile, ${ }^{17}$ the $\eta^{2}$-coordination complexes open up more complex reactivity patterns, most of which are initiated by an electrophilic attack. ${ }^{48,49}$

Early studies from Harman's group reported cycloadditions of $\eta^{2}$-coordinated Re complexes of benzene and $\mathrm{N}$-methyl pyrrolidine with reactive dienophiles to afford organic products with bicyclo[2.2.2]octane or heterocyclic bicyclo[2.2.1] heptane skeletons (Scheme 27). ${ }^{50} \mathrm{~A}$ pair of impressive studies on dearomatization of phenol via $\eta^{2}$-coordination with $\mathrm{W}$ uncovered a regio- and stereoselective $[2+2]$ reaction of the $\mathrm{W}$ complexes of phenol with ketene, ${ }^{51}$ as well as a series of selective bis-functionalizations of the complexed phenol to afford cis-4,5-disubstitued cyclohex-2-en-1-ones (Scheme 27). ${ }^{52}$ 

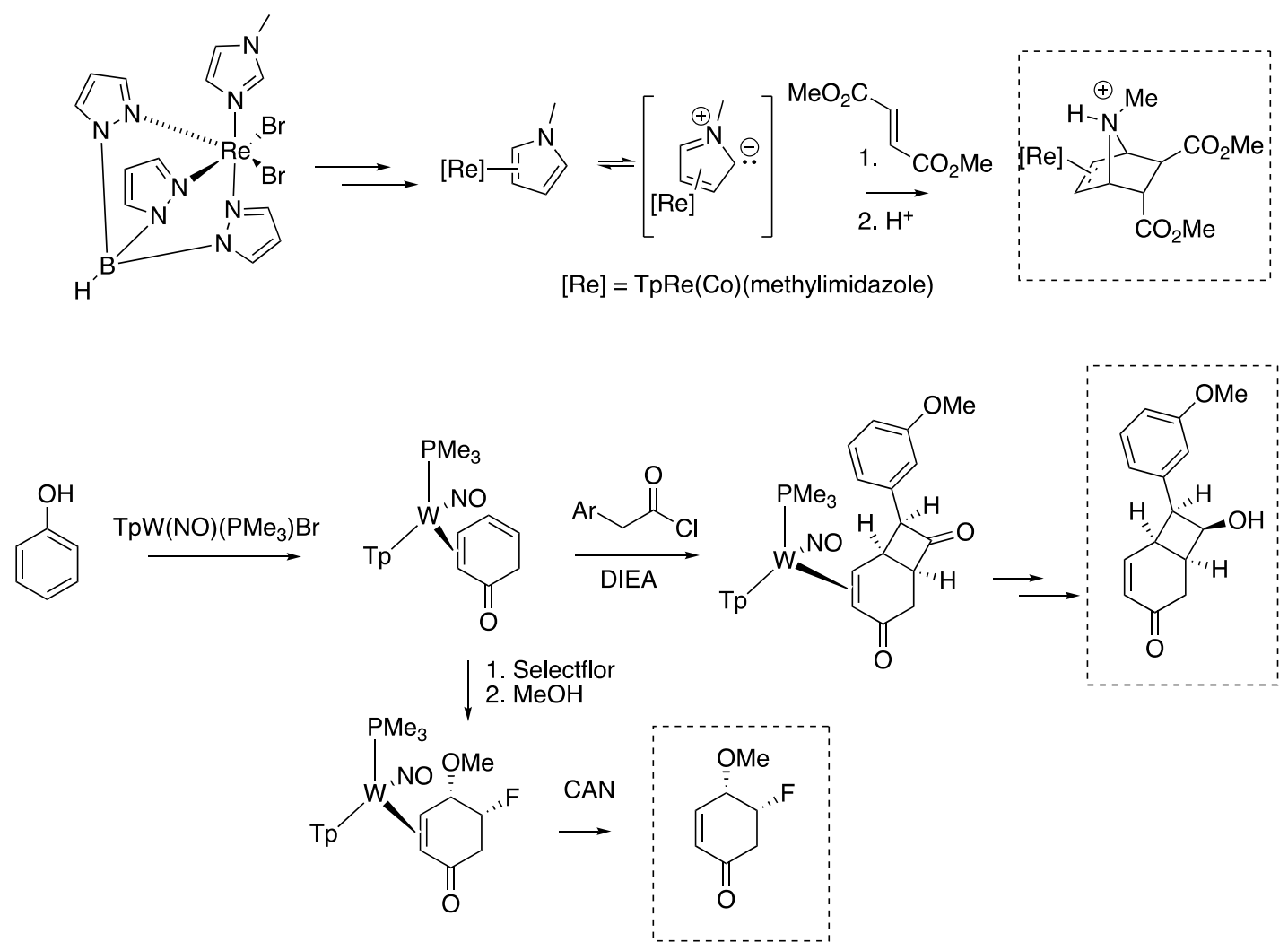

\section{Scheme 27}

Protocols for dearomatization of diverse aromatic and heteroaromatic substrates via $\eta^{2}$-coordinated group-6 (W and Mo) complexes have been reviewed, including publications through the year $2017 . .^{53}$ This review will focus on the most recent advances in applications of chiral nonracemic $\eta^{2}$-coordinated $\mathrm{W}$ and $\mathrm{Mo}$ complexes to asymmetric synthesis.

The first preparation of enantiopure tungsten complex was achieved by a classical resolution approach. ${ }^{54}$ Protonation of racemic chiral at tungsten complexes (3:1 mixture of coordination diastereomers) of electron-rich aromatic ligands with single enantiomers of chiral nonracemic acids afforded a mixture of two diastereomeric salts with differential solubility. Application of either L- or D-dibenzoyltartaric acids (DBTH2) afforded selectively either enantiomer of the $\mathrm{Tp}\left(\mathrm{PMe}_{3}\right)(\mathrm{NO}) \mathrm{W}\left(\eta^{2}\right.$-benzene) complex in high enantiomeric purities (Scheme 28). 


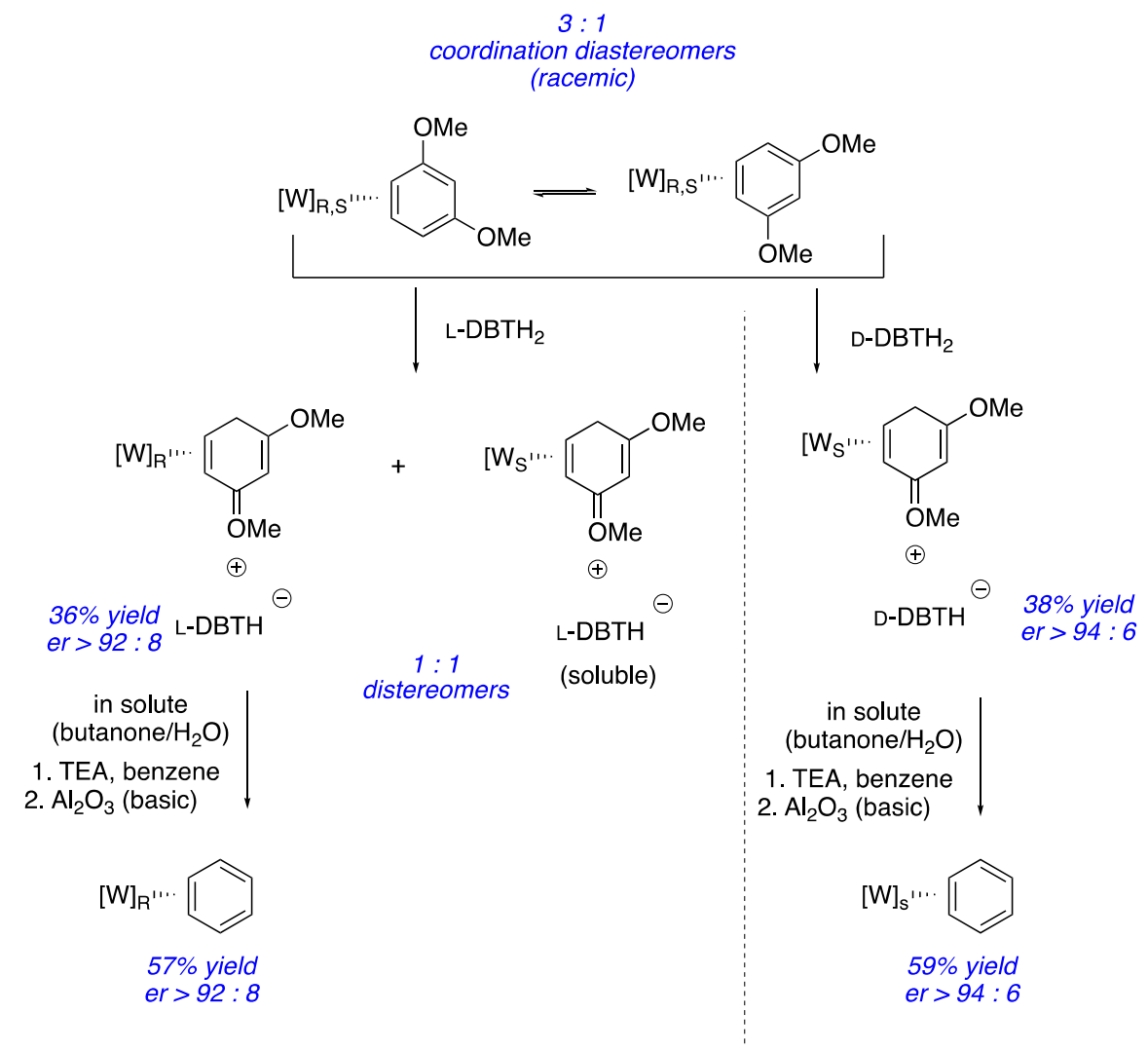

\section{Scheme 28}

In contrast to electron-deficient aromatics (e.g. trifluoromethyl benzene) that stabilize the $\eta^{2}$ complexes by significant metal to ligand $\pi$-back bonding, ${ }^{55}$ selective dearomatization of benzene, proved to be quite challenging. ${ }^{56}$ The challenge has been effectively addressed using the chiral-at-tungsten, enantiopure complexes that added sequentially first a proton, and then a nucleophile, across the uncoordinated double bond distal to the phosphine ligand, both from the face opposite to the metal fragment. After decomplexation, the protocol produced cis-disubstituted cyclohexenes in a high enantiomeric purity (Scheme 29).
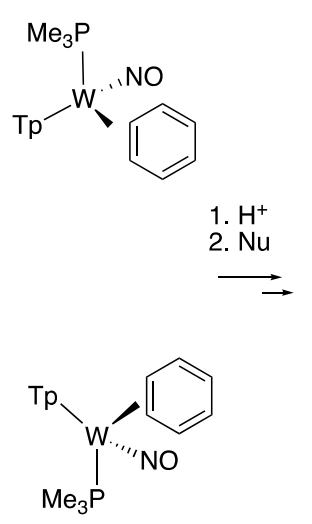

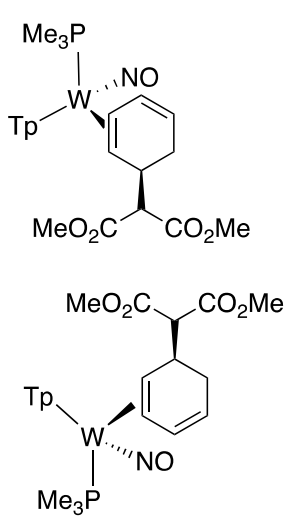

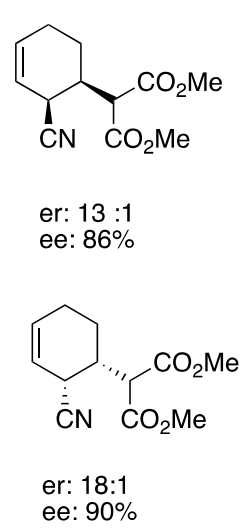

\section{Scheme 29}


Development of effective protocols for enantioselective dearomatization mediated by Mo-complexes presented a greater challenge than the prior studies with Re and W. ${ }^{54}$ The strategy employed for preparation of enantiopure chiral-at-molybdenum complexes relied on a ligand exchange between racemic, chiral at molybdenum complex and a chiral ligand ( $\alpha$-pinene) to afford a mixture of diastereomeric complexes. Following the separation of the diastereomers by classical techniques, the treatment of the isolated single diastereomers with organic substrates was expected to proceed with retention of stereochemistry at the molybdenum center and to release the $\alpha$-pinene ligand.

Towards this goal, reduction of racemic $\mathrm{Mo}(\mathrm{l})$-complex with $\mathrm{Na}$ in the presence of $(R)$ - $\alpha$-pinene afforded the $\mathrm{Mo}(0)$-complex with the chiral ligand as a single diastereomer with $\left(R_{\mathrm{Mo}}, R_{\mathrm{Lig}}\right)$ configuration (Scheme 30). ${ }^{57}$ Due to the lower $\pi$-basicity of the Mo-complex in comparison to $\operatorname{Re}$ or $W$ analogs, the mismatched ( $S_{\mathrm{Mo}}, R_{\mathrm{Lig}}$ ) diastereomer proved too unstable to be isolated. The matched $\left(R_{\mathrm{Mo}}, R_{\mathrm{Lig}}\right)$ diastereomer participated in subsequent ligand exchange reactions quite easily at ambient temperature. Disappointingly, careful studies established that isomerization at the molybdenum stereocenter took place during the ligand exchange reactions with the intended aromatic substrates, possibly via a square planar intermediate formed by a dissociative mechanism.

Reasoning that oxidation of the $\mathrm{Mo}(0)$ center to $\mathrm{Mo}(\mathrm{I})$ would minimize the racemization at the metal stereocenter, an impressive solution to this problem was designed. ${ }^{57}$ Thus, oxidative $\left(\mathrm{I}_{2}\right.$, THF) replacement of the $\alpha$-pinene ligand in the Mo(0)-complex afforded a Mo(I)-iodo complex in an excellent yield and high enantiopurity. Next, the enantioselective dearomatization of trifluoromethyl benzene was explored. The reduction (by treatment with $\mathrm{Na}$ ) of the $\mathrm{Mo}(\mathrm{I})$-iodo complex in neat trifluoromethyl benzene at $15^{\circ} \mathrm{C}$ afforded a single diastereomer of the Mo(0)-complex in good yield and high er (99: 1). Complexation of the trifluoromethyl benzene to the Mo center activated the organic substrate for a sequential regiospecific protonation, followed by nucleophilic attack by a masked enolate yielding the corresponding diene in a high enantiopurity after oxidative demetalation (Scheme 30).
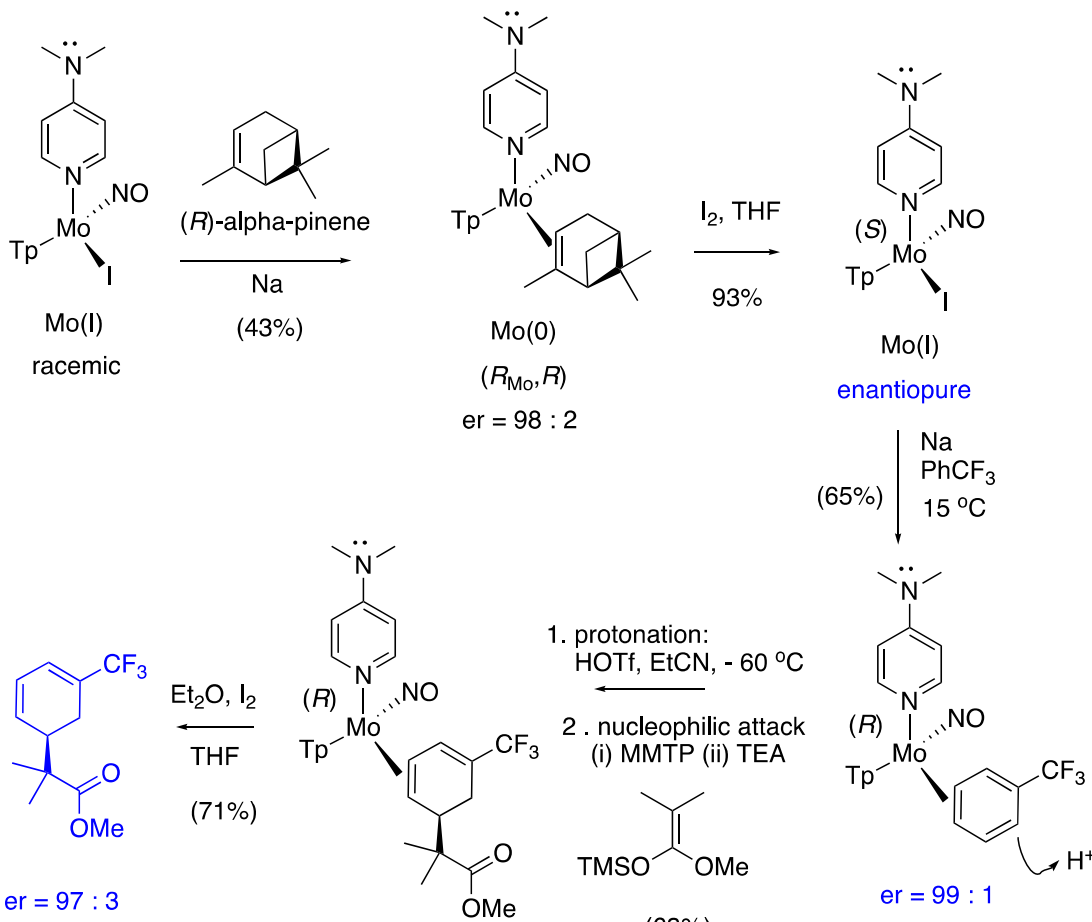

$(63 \%)$

\section{Scheme 30}


A critically important step in the evolution of asymmetric desymmetrization via $\eta^{2}$-coordinated molybdenum complexes is the extension of the methodology to the elaboration of heterocycles, particularly the N-heterocycles.

Recently, complexation of molybdenum to 2-trifluoromethyl pyridine was made possible by the stabilizing effect of an electron-withdrawing $\mathrm{CF}_{3}$ group, that also blocked the coordination of the nitrogen atom to the metal center. ${ }^{58}$ Although the initial coordination of the Mo to the heterocycle was not stereoselective, affording a $3: 2$ mixture of coordination diastereomers that rapidly equilibrated in solution, a process for the preparation of a single diastereomer of the molybdenum complex was devised. A single diastereomer was found to be strongly favored in the crystalline phase. Thus, stirring the suspension of the coordination diastereomers in acetonitrile for two hours afforded a solid phase containing a single diastereomer, which was subsequently stabilized by N-methylation (Scheme 31).
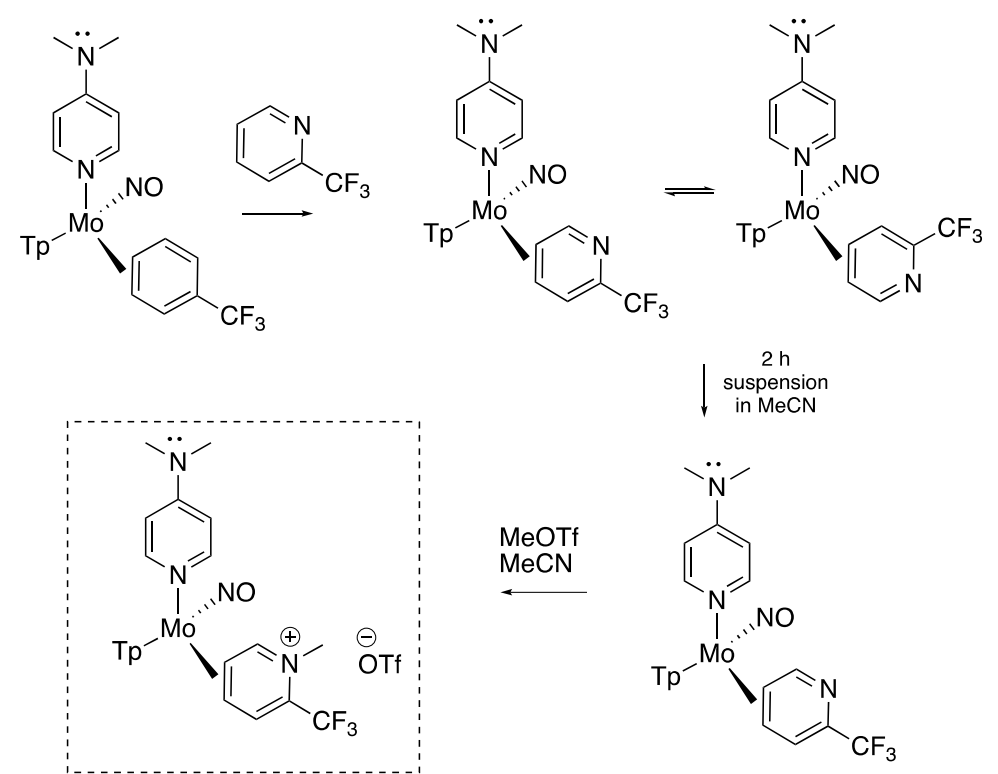

\section{Scheme 31}

The molybdenum complex with the pyridinium ligand proved to be an excellent substrate in nucleophilic addition reactions with a variety of nucleophiles. The reactions proceeded exclusively at the C- 6 position and anti to the molybdenum centre (Scheme 32). This reactivity contrasts with the preference for C-4 functionalization of traditional pyridinium salts. Analogous complexes incorporating the more explored tungsten metal centre showed the same reactivity patterns as the molybdenum counterparts but lacked the convenience of metal fragment recycling (Scheme 32).

When considering the development of an asymmetric variant of the desymmetrization of trifluoromethylpyridine, concerns about possible side reactions between the sodium metal reductant, required in the last step of the synthesis of enantiopure molybdenum complex, and the heterocycle arose. ${ }^{48}$ In the event, the key reductive ligand exchange proceeded successfully, albeit in limited yields (24\%), permitting the preparation of an enantiopure 2,6-disubstituted dihydropyridine, as well as its Diels-Alder adduct featuring the [2.2.2] bridged system of isoquinuclidine (Scheme 32). ${ }^{58}$ 

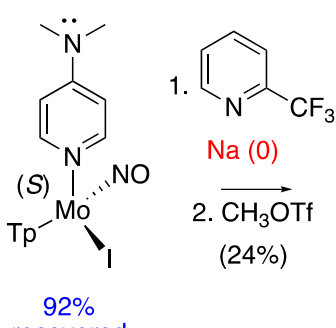

2. $\overrightarrow{\mathrm{CH}_{3} \mathrm{OTf}}$

(24\%)

recovered
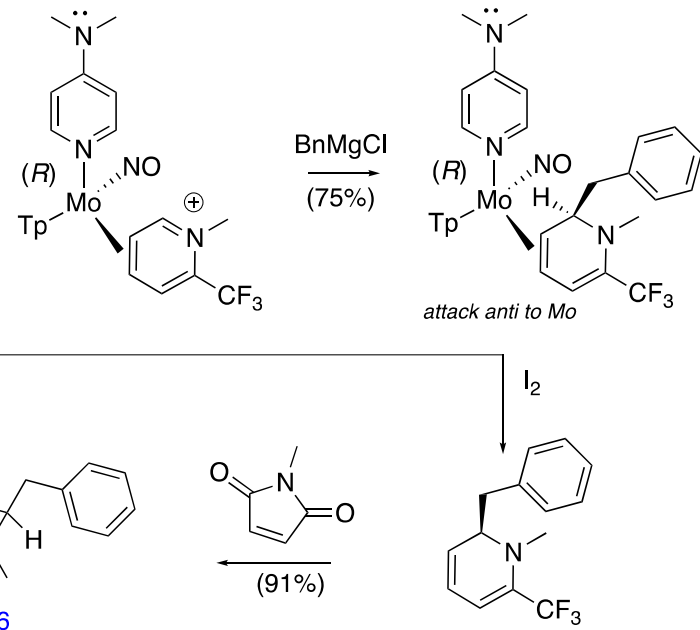

$(79 \%)$

\section{Scheme 32}

The most impressive achievement of the dearomatization methodology in terms of the complexity of bond-formation is the racemic synthesis of the complete steroidal core by a Michael-Michael ring closure reaction of the molybdenum complex with $\eta^{2}$-coordinated naphthalene (Scheme 33). ${ }^{59}$ The protocol was performed as a one-pot operation providing the organic product as a single stereoisomer in an overall $13 \%$ yield.
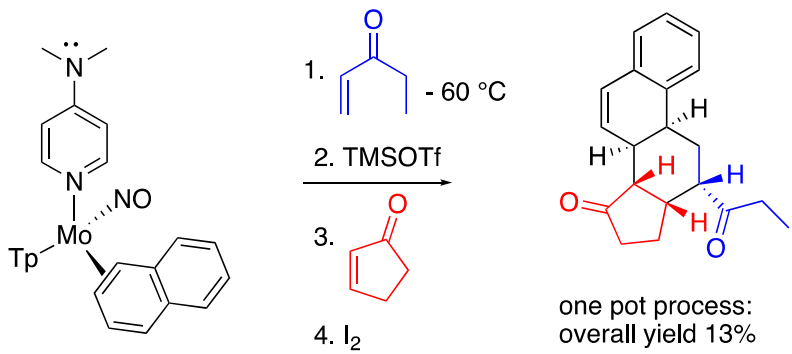

\section{Scheme 33}

\section{Conclusions}

Studies described in this review highlight the advances in asymmetric organic synthesis achieved by applications of isolable transition metal complexes. A particular emphasis was placed on methodologies exploiting stereoselective functionalization of chiral nonracemic complexes with planar chirality. ${ }^{17-49}$ Material presented herein convincingly demonstrates the value of such synthetic methodologies as practical tools for the synthesis of chiral nonracemic molecules of biological origin, including highly functionalized heterocycles. The modular and sequential nature of these processes appears uniquely suited for applications in molecular library synthesis. Furthermore, specialized methodologies for $\mathrm{C}-\mathrm{H}$ activation in stoichiometric transition metal templates may prove invaluable for selective elaborations of complex organic substrates, as well as to provide fundamental insights relevant to the development of catalytic transformations. ${ }^{5-16}$ 


\section{References}

1. Kauffman, G. B. J. Chem. Educ. 1983, 60, 3, 185.

https://doi.org/10.1021/ed060p185

2. Heck, R. F. J. Am. Chem. Soc. 1969, 91, 6707.

https://doi.org/10.1021/ja01052a029

3. Churchill, M. R.; Ziller, J. W.; Freudenberger, J. H.; Schrock, R. R. Organometallics 1984, 3, 1554. https://doi.org/10.1021/om00088a018

4. All Nobel Prizes in Chemistry. https://www.nobelprize.org/prizes/lists/all-nobel-prizes-in-chemistry/ (accessed 12/14/2020).

5. Semmelhack, M. F.; Clark, G. R.; Garcia, J. L.; Harrison, J. J.; Thebtaranonth, Y.; Wulff, W.; Yamashita, A. Tetrahedron 1981, 37, 3957. https://doi.org/10.1016/S0040-4020(01)93270-3

6. Dotz, K. H.; Kuhn, W. Angew. Chem. Int. Ed. Engl. 1983, 22, 732. https://doi.org/10.1002/anie.198310450

7. Yamamoto, J.; Kawashima, A.; Kawamura, A.; Abe H.; Moriwaki, H.; Shibata, N.; Soloshonok, V. A. Eur. J. Org. Chem. 2017, 1931.

https://doi.org/10.1002/ejoc.201700018

8. Mkrtchyan, A. F.; Hayriyan, L. A.; Karapetyan, A. J.; Tovmasyan, A. S.; Tsaturyan, A. H.; Khrustalev, V. N.; Maleev, V. I.; Saghyan, A. S. New J. Chem. 2020, 44, 11927.

https://doi.org/10.1039/d0nj02072a

9. Zhao, C.; Crimmin, M. R.; Toste, F. D.; Bergman, R. G. Acc. Chem. Res. 2014, 47, 517. https://doi.org/10.1021/ar400176x

10. Boyd, W. C.; Crimmin, M. R.; Rosebrugh, L. E.; Shomaker, J. M.; Bergman, R. G.; Toste, F. D. J. $\quad A m$. Chem. Soc. 2010, 132, 16365. https://doi.org/10.1021/ja107968c

11. Zhao, C.; Toste, F. D.; Bergman, R. G. J. Am. Chem. Soc. 2011, 133, 10787. https://doi.org/10.1021/ja204564z

12. Sodeoka, M.; Shibasaki, M. Synthesis 1993, 643. https://doi.org/10.1055/s-1993-25917

13. da Costa, M. R. G.; Curto, M. J. M.; Davies, S. G.; Teixeira, F. C.; Thomson, J. E. Tetrahedron 2017, $73,5411$. http://dx.doi.org/10.1016/j.tet.2017.07.039

14. McGrew, G. I.; Stanciu, C.; Zhang, J.; Carroll, P. J.; Dreher, S. D.; Walsh, P. J. Angew. Chem. Int. Ed. 2012, 51, 11510.

https://doi.org/10.1002/anie.201201874

15. Takemoto, S.; Matsuzaka, H. Tetrahedron Lett. 2018, 59, 697. https://doi.org/10.1016/i.tetlet.2018.01.036

16. Oestreich, M.; Dennison, P. R.; Kodanko, J. J.; Overman, L. E. Angew. Chem. Int. Ed. 2001, 40, 1439. https://doi.org/1433-7851/01/4008-1440

17. Burke, B. J.; Overman, L. E. J. Am. Chem. Soc. 2004, 126, 16820. https://doi.org/10.1021/ja045047p

18. Lu, G.; Malinakova, H. C. J. Org. Chem. 2004, 69, 4701. https://doi.org/10.1021/jo040148r 
19. Pape, A. R.; Kaliappan, K. P.; Kundig, E. P. Chem. Rev. 2000, 100, 2917. https://doi.org/10.1021/cr9902852

20. Kundig, E. P.; Cannas, R.; Laxmisha, M.; Ronggang, L.; Tchertchian, S. J. Am. Chem. Soc. 2003, $125,5642$. https://doi.org/10.1021/ja029957n

21. Pigge, C. F.; Coniglio, J. J.; Rashmi, D. J. Am. Chem. Soc. 2006, 128, 3498.

https://doi.org/10.1021/ja058342y

22. Pearson, A. J.; Sun, H.; Wang, X. J. Org. Chem. 2007, 72, 2547.

https://doi.org/10.1021/jo0625608

23. Knölker, H.-J.; Baum, E.; Kosub, M. Synlett 2004, 1769.

https://doi.org/10.1055/s-2004-829545

24. Stephenson, R. G.; Roe, C.; Anson, C. E. J. Org. Chem. 2012, 77, 9684.

https://doi.org/doi.org/10.1021/jo301617f

25. Stephenson, R. G.; Roe, C.; Sandoe, E. J. Eur. J. Org. Chem. 2011, 1664. https://doi.org/10.1002/ejoc.201001394

26. Roe, C.; Sandoe, E. J.; Stephenson. R. G.; Anson, C. E. Tetrahedron Lett. 2008, 49, 650. https://doi.org/10.1016/j.tetlet.2007.11.137

27. ten Broeke, M.; Khan, M. A.; Kociok-Kohn, G.; Kann, N.; Lewis, S. E. J. Organomet. Chem. 2015, 19-20, 799. http://dx.doi.org/ 10.1016/j.jorganchem.2015.09.005

28. Palframan, M. J.; Kociok-Kohn, G.; Simon E. Lewis, S. E. Org. Lett. 2011, 13, 3150. https://doi.org/10.1021/ol201057r

29. Hansson, S.; Miller, J.; Liebeskind, L. S. J. Am. Chem. Soc. 1990, 112, 9660. https://doi.org/10.1021/ja00182a047

30. Moretto, A. F.; Liebeskind, L. S. J. Org. Chem. 2000, 65, 7445. https://doi.org/10.1021/jo0007128

31. Adams, R. D.; Chodosh, D. F.; Faller, J. W.; Rosan, A. M. J. Am. Chem. Soc. 1979, 10, 2570. https://doi.org/10.1021/ja00504a012

32. Faller, J. W.; Murray, H. H.; White, D. L.; Chao, K. H. Organometallics 1983, 2, 400. https://doi.org/10.1021/om00075a009

33. Shu, C.; Alcudia, A.; Yin, J.; Liebeskind, L. S. J. Am. Chem. Soc. 2001, 123, 12477. https://doi.org/10.1021/ja011635g

34. Yin, J.; Llorente, I.; Villanueva, L. A.; Liebeskind, L. S. J. Am. Chem. Soc. 2000, 122, 10458. https://doi.org/10.1021/ja002694h

35. Malinakova, H. C.; Liebeskind, L. S. Org. Lett. 2000, 2, 3909. https://doi.org/10.1021/ol000288x

36. Shu, C.; Liebeskind, L. S. J. Am. Chem. Soc. 2003, 125, 2878. https://doi.org/10.1021/ja029537y

37. Wong, H.; Garnier-Amblard, E. C.; Liebeskind, L. S. J. Am. Chem. Soc. 2011, 133, 7517. https://pubs.acs.org/doi/10.1021/ja201012p

38. Zhang, Y.; Liebeskind, L. S. J. Am. Chem. Soc. 2006, 128, 465. https://doi.org/10.1021/ja055623x

39. Gomez-Arrayas, R.; Liebeskind, L. S. J. Am. Chem. Soc. 2003, 125, 9026. https://doi.org/10.1021/ja035424i

40. Gomez-Arrayas, R.; Yin, J.; Liebeskind, L. S. J. Am. Chem. Soc. 2007, 129, 1816. https://doi.org/10.1021/ja067104f 
41. Coombs, T. C.; Lee, M. D. IV; Wong, H.; Armstrong, M.; Cheng, B.; Chen, W.; Moretto, A. F.; Liebeskind, L. S. J. Org. Chem. 2008, 73, 882. https://doi.org/10.1021/jo702006z

42. Garnier, E. C.; Liebeskind, L. S. J. Am. Chem. Soc. 2008, 130, 7449. https://doi.org/10.1021/ja800664v

43. Cheng, B.; Liebeskind, L. S. Org. Lett. 2009, 11, 3682. https://doi.org/10.1021/ol901523g

44. Zhang, Y.; Liebeskind, L. S. J. Am. Chem. Soc. 2005, 127, 11258. https://doi.org/10.1021/ja0538132

45. Coombs, T. S.; Zhang, Y.; Garnier-Amblard, E. C.; Liebeskind, L. S. J. Am. Chem. Soc. 2009, 131, 876. https://doi.org/10.1021/ja808533z

46. Chen, W.; Sana, K.; Jiang, Y.; Meyer, E. V. S.; Lapp, S.; Galinski, M. R.; Liebeskind, L. S. Organometallics 2013, 32, 7594. https://doi.org/10.1021/om401087h

47. Chen, W.; Liebeskind, L. S. J. Am. Chem. Soc. 2009, 131, 12546. https://doi.org/10.1021/ja9056322

48. Harman, W. D. Chem. Rev. 1997, 97, 1953. https://doi.org/10.1021/cr940316n

49. Chen, H.; Harman, W. D. J. Am. Chem. Soc. 1996, 118, 5672. https://doi.org/10.1021/ja960389j

50. Chordia, M. D.; Smith, P. L.; Meiere, S. H.; Sabat, M.; Harman, W. D. J. Am. Chem. Soc. 2001, $123,10756$. https://doi.org/10.1021/ja011689q

51. Todd, M. A.; Sabat, M.; Myers, W. H.; Harman, W. D. J. Am. Chem. Soc. 2007, 129, 11010. https://doi.org/10.1021/ja0739279

52. Todd, M. A.; Sabat, M.; Myers, W. H.; Smith, T. M.; Harman, W. D. J. Am. Chem. Soc. 2008, $130,6906$. https://doi.org/10.1021/ja801076z

53. Liebov, B. K.; Harman, W. D. Chem. Rev. 2017, 117, 13721. https://doi.org/10.1021/acs.chemrev.7b00480

54. Lankenau, A. W.; Iovan, D. A.; Pienkos, J. A.; Salomon, R. J.; Wang, S.; Harrison, D. P.; Myers, W. H. Harman, W. D. J. Am. Chem. Soc. 2015, 137, 3649. https://doi.org/10.1021/jacs.5b00490

55. Myers, J. T.; Smith, J. A.; Dakermanji, S. J.; Wilde, J. H.; Wilson, K. B.; Shivokevich, P. J.; Harman, W. D. J. Am. Chem. Soc. 2017, 139, 11392. https://doi.org/10.1021/jacs.7b05009

56. Wilson, K. B.; Smith, J. A.; Nedzbala, H. S.; Pert, E. K.; Dakermanji, S. J.; Dickie, D. A.; Harman, W. D. J. Org. Chem. 2019, 84, 6094. https://doi.org/10.1021/acs.joc.9b00279

57. Shivokevich, P. J.; Myers, J. T.; Smith, J. A.; Pienkos, J. A.; Dakermanji, S. J.; Pert, E. K.; Welch, K. D.; Trindle, C. O.; Harman, W. D. Organometallics 2018, 37, 4446. https://doi.org/10.1021/acs.organomet.8b00027

58. Wilde, J. H.; Smith, J. A.; Dickie, D. A.; Harman, W. D. J. Am. Chem. Soc. 2019, 141, 18890. https://doi.org/10.1021/jacs.9b10781

59. Myers, J. T.; Wilde, J. H.; Sabat, M.; Dickie, D. A.; Harman, D. W. Organometallics 2020, 39, 1404. https://dx.doi.org/10.1021/acs.organomet.0c00110 


\section{Authors' Biographies}

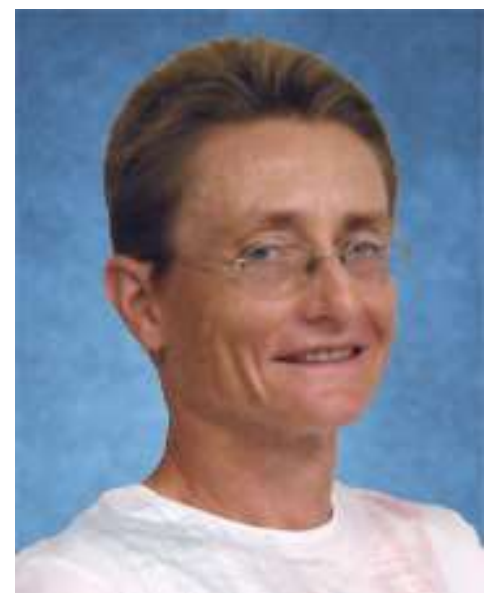

After a Master Degree in chemistry from the Institute of Chemical Technology in Prague (Czech Republic) (1989), Helena Malinakova received her Ph.D. (Illinois Institute of Technology, Chicago, IL) in 1998 for studies on palladium-catalyzed reactions for the synthesis of antitumor quinone natural products. Then she joined Prof. Lanny S. Liebeskind's group at Emory University (Atlanta, Georgia), as a postdoctoral fellow in 1999 2000 working on the development of methods for asymmetric synthesis of $\mathrm{N}$-heterocycles using chiral nonracemic complexes of molybdenum. In 2000, she joined the faculty at the University of Kansas, where she has been pursuing a research program exploring the chemistry of palladium(IV) complexes, as well as development of transition metal-catalyzed sequential one-pot and multicomponent reactions for the synthesis of heterocycles. 